Article

\title{
Overcoming Challenges in Street Art Murals Conservation: A Comparative Study on Cleaning Approach and Methodology
}

\author{
Moira Bertasa ${ }^{1}$, Chiara Ricci ${ }^{2}$, Arianna Scarcella ${ }^{2}$, Francesca Zenucchini ${ }^{2}$, Giulia Pellis ${ }^{1}$, \\ Paola Croveri $^{2}$ and Dominique Scalarone ${ }^{1, * \mathbb{D}}$ \\ 1 Department of Chemistry, University of Torino, Via Pietro Giuria 7, 10125 Torino, Italy; \\ moira.bertasa@unito.it (M.B.); giulia.pellis@unito.it (G.P.) \\ 2 Fondazione Centro Conservazione e Restauro "La Venaria Reale", 10078 Venaria Reale, Italy; \\ chiara.ricci@centrorestaurovenaria.it (C.R.); arianna.scarcella@centrorestaurovenaria.it (A.S.); \\ francesca.zenucchini@centrorestaurovenaria.it (F.Z.); paola.croveri@centrorestaurovenaria.it (P.C.) \\ * Correspondence: dominique.scalarone@unito.it; Tel.: +39-011-670-7546
}

Received: 29 September 2020; Accepted: 21 October 2020; Published: 23 October 2020 updates

\begin{abstract}
Street art, and its preservation, has become one of the most discussed and still open topics in the field of conservation. The absence of a sharable conservation protocol in terms of cleaning and protection is a delicate subject to deal with. The cleaning of contemporary murals and urban artworks stained by graphical vandalism is a real challenge, due to the similar chemical composition of the materials involved. In this work two different approaches to the cleaning of street art murals, namely chemical methods and laser cleaning, are reported and compared with the aim of identifying a suitable conservation practice. Ad hoc concrete mock-ups coated with a paint layer and a simulated vandalism on top have been prepared and used for the testing and analytical assessment of the cleaning procedures.
\end{abstract}

Keywords: street art; graffiti; cleaning; solvent; gel; laser; conservation; paint; spray

\section{Introduction}

In the last decade, the definition of scientific approaches to street art murals conservation is one of the main topics strongly discussed in the field of conservation [1-5]. The lack of shared conservative guidelines on this form of contemporary art, as well as a clear legislation, represents a great problem for conservators, who are more and more often involved on this subject.

Currently, street art is no more considered as only an illegal outcome of social discomfort, but a decorative, didactic and commemorative representation, often advocate of collective reflection on topics of social interest, enough to be promoted by municipalities and galleries. Indeed, as reported by the literature $[1,6]$, two distinct groups of contemporary artistic expression can be outlined: (I) the so-called post-graffiti interventions, such as spontaneous and illegal artistic expressions, created by the artists, and (II) the commissioned interventions promoted into festivals and institutional projects to dignify degraded urban areas with agreed subjects or to involve citizens in a sort of public "graphic reflection" on current topics (see "TO-ward 2030 What are you doing" project, supported by Turin Municipality). In this context, it is clear how different purposes and different artistic conceptions of the final subject involve distinct overtures in terms of conservation approach. Equally, what is nowadays defined as graphical vandalism is considered as a threat for the conservation of historical buildings and sculptures, as well as for commissioned art murals once they have been stained. For this reason, a proper cleaning approach appears to be essential within this framework. 
It is noteworthy that the materials used in these unwanted graphical expressions are often similar and, in many cases, are also chemically equal to the constitutive materials of the artworks themselves. The range of materials adopted in contemporary art constantly expands and in general, the main chemical classes include alkyd, acrylic, styrene resins and their combinations [7-16]. Thus, it is pretty clear how the physical or chemical cleaning of stained murals might become even more delicate and complex.

In the recent years, the application of protective coatings has been discussed focusing the attention on the physico-chemical properties of the products themselves and on any possible interaction with the constitutive materials. Specific protective coatings, called "anti-graffiti", can be classified in sacrificial, semi-sacrificial and permanent coatings on the basis of their "durability" on the treated surface $[17,18]$. The selection of one or the other depends on a lot of factors, ranging from the environmental boundary conditions to the feasibility of carrying out a regular maintenance of the artworks. The literature reports several research projects aimed to identify proper anti-graffiti products for the protection of the bare architectural surfaces [19-21], while fewer works are available about the use of anti-graffiti on contemporary murals [22]. Indeed, Macchia's research proposes the application of the same anti-graffiti materials commonly used on stone as protective coatings also against graphical vandalism. Furthermore, a biocleaning approach for the removal of graffiti paints has recently been evaluated by testing an efficient biocleaning protocol for in situ applications [23-25].

Envisaging a growing demand to preserve or even restore these types of works of art, it is necessary to develop new cleaning approaches, aimed to share successful practices and define widely useful criteria both for products/methodologies definition and for scientific evaluation of the results. These approaches, other to be applied to unconventional materials, such as spray paints, must also take into account the increasingly pressing requests in terms of environment protection and sustainability [26]. Cleaning of artworks is a critical phase and it is required to be highly controllable, selective, efficient and safe for the environment and the operator. In the last twenty years, new researches have been undertaken to reduce the environmental impact and promote depollution. This concept is well known as green chemistry and in some way, it became part of the conservation practice with the use of the so-called green solvents [27-30], but the limits due to the use of harmful substances make in practice an ideal cleaning quite complicated. The will to reach as much as possible these features, especially when cleaning operations should be carried out outdoor, within an urban space as in the case of street art, is the motivation to propose streamlined and feasible cleaning approaches.

This paper reports on a comparative study for the identification of a proper cleaning method in removing unwanted painting materials on ad hoc specimens. Both chemical and laser cleaning were tested on mock-ups representing the most common materials encountered in street art. Simulating a real mural artwork stained by graphical vandalism, four different stratigraphy with overlapping styrene-acrylic paints and alkyd paints have been reproduced in the laboratory. In particular, two different contrasting colors, such as red and green, have been intentionally selected to make as easy as possible the visual observation of the cleaning test results. Furthermore, imitating a potential conservation treatment, a protective coating has been applied on half portion of each mock-up, between the first and the second paint layer. The choice of the protective coating fell to a waterproof material already applied on the contemporary mural Tutto Mondo, by Keith Haring, in a recent conservation intervention [31].

The aim of this research was to compare and evaluate with various analytical techniques the effectiveness and selectivity of different cleaning methods with low or no environmental impact to remove the outermost paint layer, which simulates a hypothetical vandalism. The experimental plan applied in this research, and described by the flow chart shown in Figure 1, consists of an initial characterization of the paints, to identify the chemical class they belong to, and subsequently a series of analysis with increasing sensitivity to detect residues of vandalism after cleaning, starting with macroscopic observation, to continue with microscope investigations, colorimetry, FTIR and finally Py-GC/MS. 


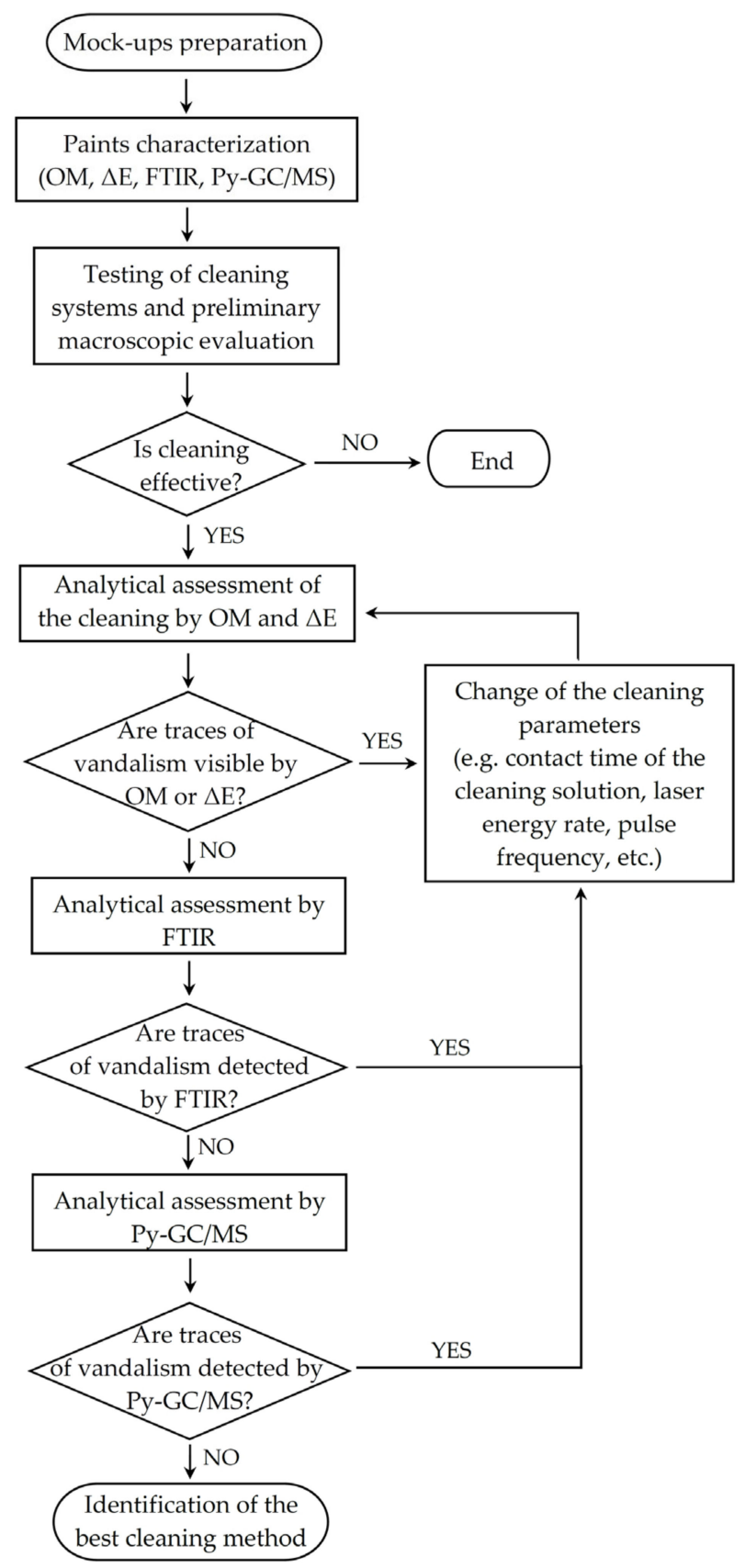

Figure 1. Flow chart of the experimental plan. OM: optical microscopy; $\Delta E$ : colorimetric measurements.

Overall, this study could be an invaluable tool for developing future street art conservation practices.

\section{Materials and Methods}

\subsection{Materials}

\subsubsection{Mock-ups Preparation}

Four different stratigraphies were prepared on concrete specimens. According to the preliminary survey of the most common supports for street art murals in Turin, concrete was chosen as a significant substrate. The mock-ups were prepared with i.work TECNOCEM 32,5 R by ITALCEMENTI, a Portland concrete with limestone type II, characterized by a strong resistance in the drying phase. The product, 
according to the UNI EN $197-1$ standard, contains $80 \%$ up to $94 \%$ of clinker, while the remaining fraction consists of limestone (TOC $\leq 0.20 \%$, in mass (LL)) and eventually other minor constituents. In order to realize the mock-ups, the Portland concrete was added with local river sand in the ratio 1:3 and then mixed manually, adding water to facilitate the processing of the mortar.

In order to obtain similar dimensions and shapes, silicone molds were used, as a result all the mock-ups measure $8 \times 5.5 \mathrm{~cm}$, with a thickness of $2 \mathrm{~cm}$. In order to be sure of the complete drying of the mortar, the mock-ups were left to air-dry for two months. Two replicas for each stratigraphy were prepared and used to test chemical and laser cleaning methods, respectively. Table 1 reports all the possible stratigraphies obtained by applying on each mock-up two paint layers, selected as appropriate among four different paints, respectively two styrene-acrylic paints and two alkyd paints. These were considered as representative examples of the most common categories of painting materials found in real street artworks. Two layers of the styrene-acrylic paints were applied by brush, with a water dilution of $30 \% v / v$, as suggested by the supplier technical data sheets; before applying the second painting layer, a drying time of $14 \mathrm{~h}$ (in laboratory conditions, $20 \pm 2{ }^{\circ} \mathrm{C}$ and $50 \pm 10 \% \mathrm{RH}$ ) has been respected. Instead, the alkyd paints were sprayed on the surface from a distance of about $20-30 \mathrm{~cm}$ and were overpainted after $30 \mathrm{~min}$. The top layer was applied after three months. After the application of the second paint layer, samples were left to air-dry in laboratory conditions for ten days. The application of the top painting layers follows the same methodology previously described: also in this case, two level for both styrene-acrylic and alkyd paints were applied.

Table 1. Specimens stratigraphy.

\begin{tabular}{ccc}
\hline \multirow{2}{*}{ Mock-up Name } & \multicolumn{2}{c}{ Stratigraphy } \\
\cline { 2 - 3 } & Top Layer & Bottom Layer \\
\hline A & Red styrene-acrylic paint & Green styrene-acrylic paint \\
B & Red alkyd spray paint & Green alkyd spray paint \\
C & Red styrene-acrylic paint & Green alkyd spray paint \\
D & Green alkyd spray paint & Red styrene-acrylic paint \\
\hline
\end{tabular}

On each mock-up, two contrasting colors were applied to ease the assessment of the selectivity of the cleaning procedure even by visual observation during the cleaning operation. In particular, Military green (n. \#173 Evil Olive) and red (n. \#016-SWET 100 Traffic Red) for the alkyd spray paint, purchased by Molotov Premium Belton, and green (color code G8.40.40) and red (color code C6.65.35) Alpha Acrilmat by Sikkens for the acrylic brush paint were respectively selected. Between one color and another, the SILO 112 protective coating (CTS s.r.l., Altavilla Vicentina, Italy) was applied on half portion of each specimen to evaluate its contribution to the efficiency of the cleaning method.

\subsubsection{Cleaning Materials and Method}

The cleaning tests were carried out with chemical and laser cleaning methodologies, ten days after the application of the bottom layer, with the aim of investigating an early cleaning intervention on a mural. In particular, in the framework of the traditional chemical cleaning methods, a wide range of thickeners and solvents were evaluated by means of preliminary tests with the aim to identify a limited number of promising formulations to test more in detail on the specimens reported in Table 1. Ethyl cellulose, gellan and poly(vinyl alcohol)-borate gel were considered, likewise several solvents, such as water, isopropyl alcohol, acetone, MEK or diethyl carbonate, just to name a few. A preliminary evaluation of the interactions with the paints and the effectiveness of the cleaning has been made optically, during the application of the solvents by cotton swab or in gels. In particular, solvents with high value of polarity, like MEK or acetone, were found to be heavily aggressive toward styrene-acrylic paints, even when applied with thickeners to reduce the penetration into the painting layers. Moreover, diethyl carbonate showed a very poor selectivity in the removal of both the styrene-acrylic paint from the alkyd one and of the alkyd from the styrene-acrylic paint; even worse results were obtained with 
the cleaning of acrylic-on-acrylic and alkyd-on-alkyd mock-ups, where a minimum application of the solvent caused surface whitening and the complete solubilization of both red and green painting layers. Water application with micro swab showed little results in the styrene-acrylic paint removal. In any case, this procedure was excluded because of the possible role of water on acrylic paints affected by exudation of surfactants and the consequent reduction of the mechanical properties of the painting layer [32].

Following these preliminary tests, the three chemical cleaning systems reported in Table 2 were selected and assessed in depth. One chemical methodology, namely the Polar Coating S (added with 9\% of Ethyl Acetate) applied with Extra Dry Nanorestore Gel ${ }^{\circledR}$ for $1 \mathrm{~h}$ and then rinsed with a water micro-swab, was tested on all the mock-ups in order to obtain comparable results. Then, a second method was selected for each stratigraphy on the basis of the preliminary tests: because of the promising results achieved with a micro-swab application of propylene carbonate, this methodology was repeated on the three mock-ups B, C and D to remove the alkyd paint from the alkyd bottom layer and the styrene-acrylic paint from the alkyd substrate respectively. Considering that the previous tests highlighted a quite positive result with the use of an ethanol-water solution for the removal of the styrene-acrylic layer from a chemically similar substrate, this methodology was also tested on mock-ups A for the same purpose. Replicating the methodology used in the preliminary tests, the 30:70 ethanol:water solution was thickened in an agar gel, directly applied on the surface for five minutes and finally rinsed with the same solution. Since the ethanol-water solution resulted to be effective for styrene-acrylic solubilization, but was responsible for a visible gloss variation on the alkyd paint, it was decided to avoid the application on mock-up C, even if it would have been suitable for the removal of the top styrene-acrylic paint.

Table 2. List of the cleaning systems used.

\begin{tabular}{ccc}
\hline Cleaning Code & Cleaning System & Supplier \\
\hline I & Propylene carbonate, anhydrous & Sigma Aldrich \\
\hline II & $\begin{array}{c}2 \text { g/L agar in water gel }+10 \text { mL of } \\
\text { 30\% ethanol in water solution }\end{array}$ & $\begin{array}{c}\text { CTS S.r.l., Italy } \\
\text { Sigma Aldrich }\end{array}$ \\
\hline III & $\begin{array}{c}\text { Extra Dry Nanorestore Gel }{ }^{\circledR} \\
+ \text { Polar Coating S } \\
+9 \% \text { ethyl acetate }\end{array}$ & $\begin{array}{c}\text { CSGI, University of Florence } \\
\text { CSGI, University of Florence }\end{array}$ \\
\hline IV & $\begin{array}{c}\text { EOS 1000 Long-Q-Switch mode } \\
\text { (LQS) Nd:YAG laser }\end{array}$ & El.En. Group, Florence, Italy \\
\hline
\end{tabular}

Furthermore, on the basis of a previous research carried out by some of the authors on a similar topic [33], an EOS 1000 Long-Q-Switch mode (LQS) Nd:YAG laser system was selected (Table 2). This laser equipment (El.En., Calenzano, Italy) works at wavelength $1064 \mathrm{~nm}$, with a user-selectable energy rate at $130 \mathrm{~mJ}$ (LQS-I), $250 \mathrm{~mJ}$ (LQS-II) and $380 \mathrm{~mJ}$ (LQS-III). The laser pulse duration is $100 \mathrm{~ns}$ with 1 to $20 \mathrm{~Hz}$ as repetition rate. A uniform removal of the painting layer is afforded by the homogeneous beam profile, a variable focus handpiece, and a beam spot size from 1 to $6 \mathrm{~mm}$.

In this work, various settings and fluences were used to assess the effectiveness in removing selectively the unwanted overpainting layer, operating with the wavelength of $1064 \mathrm{~nm}$ and an energy rate $75 \mathrm{~mJ}$ (LQS-I with reducing filter), $130 \mathrm{~mJ}$ (LQS-I) to $250 \mathrm{~mJ}$ (LQS-II). The used pulse duration is $100 \mathrm{~ns}$ pulses, with $2-8 \mathrm{~Hz}$ as repetition rate. The beam spot size used varied from 1.5 to $4 \mathrm{~mm}$. The laser cleaning efficacy were assessed in dry and wet condition. In this latter case, a Melynex ${ }^{\circledR}$ polyester film was used to avoid the rapid evaporation of the water, used as a wetting agent.

For both chemical and laser cleaning, a preliminary evaluation of the results was made, on the basis of the expertise of the conservators, evaluating the capability to selectively remove the top painting layer and the presence of any interaction with the bottom painting layer. Then, the analytical 
procedure for the evaluation of the chemico-physical properties of the cleaned surfaces described in Figure 1 was applied.

Hereinafter, each cleaning area will be labelled as follows: A, B, C, and D to identify the stratigraphy, I, II, III or IV for the cleaning systems and "wp" or "np" for the area with or without the protective coating, respectively. The "dry" or "wet" condition of the surface is also specified at the end of the name.

For chemical cleaning, the necessary precautions in terms of health and safety of the operator were taken into consideration. In particular, solvents were manipulated with the adequate PPE and following the TLV-TWA guidelines (threshold limit value and time-weighted average), where available (TLV-TWA: 1000 and 200 ppm for ethanol and Extra Dry Nanorestore Gel ${ }^{\circledR}+$ Polar Coating S, respectively). Furthermore, appropriate safety controls and a proper risk assessment were put in place to reduce the level of risk in using the laser equipment.

\subsection{Characterization}

\subsubsection{Optical Microscopy}

The mock-ups were photographed under a stereoscopic optical microscope (Olympus, Tokyo, Japan) OLYMPUS SZ X10 interfaced with a digital camera OLYMPUS Color View I. A representative fragment for each mock-up was sampled and then embedded in transparent epoxy resin; the cross section was grinded and polished to obtain a flat mirror surface to be observed and studied. The cross section was observed under VIS light and the acquisition and the elaboration of the images was done with the analySIS Five software. Preliminary analyses of the cross-sections were performed also by scanning electron microscopy ZEISS EVO60 (Carl Zeiss, Oberkochen, Germany) equipped with a microprobe EDX Bruker Quantax 200, (Bruker, Billerica, MA, USA), however the observations were not fully effective since the paint layers with the same binder and filler, but with different color, were not clearly discernible. For this reason, any further morphological characterization with this technique was not performed.

\subsubsection{Colorimetric Measurements}

Colorimetric measurements were performed with a Portable Spectrophotometers (Konica Minolta, Chiyoda, Tokyo, Japan) CM-700d KONICA Minolta in SCI (specular component included) mode; a total of 15 measurements ( 5 points of measure, 3 measurements for each point) were acquired for each cleaning test area, then the average values were calculated. The change of color $(\Delta E)$ was calculated for each area comparing color coordinates $L^{*}, a^{*}, b^{*}$ respectively obtained before and after the cleaning test [34].

\subsubsection{Attenuated Total Reflection-Fourier Transform Infrared Spectroscopy}

The infrared measurements carried out to assess the cleaning efficacy were performed with a Perkin Elmer Spectrum 100 FT-IR spectrometer (Waltham, MA, USA) equipped with a Universal ATR Sampling Accessory for FTIR-ATR (attenuated total reflection) characterization. All the spectra were collected in the wavenumber range of $650-4000 \mathrm{~cm}^{-1}$, with a resolution of $4 \mathrm{~cm}^{-1}$ and $16 \mathrm{scans} / \mathrm{spectrum}$. Sampling was carried out by using a scalpel and taking care to remove only the most superficial part of the sample. Analyses were done in triplicate collecting three samples on different spots of the same area. Each analysed area was $5 \mathrm{~mm}^{2}$.

\subsubsection{Pyrolysis-Gas Chromatography/Mass Spectrometry (Py-GC/MS)}

The painting materials and the cleaning efficacy were also assessed by Py-GC/MS analyses. Samples were derivatized with the Thermally Assisted Hydrolysis and Methylation method (THM) using tetramethylammonium hydroxide (TMAH) in aqueous solution at a concentration of $25 \%$ by weight (Sigma-Aldrich, Milan, Italy). A micro-furnace Multi-Shot Pyrolyzer EGA/Py-3030D 
(Frontier Lab, Koriyama, Fukushima, Japan) coupled to a GC/MS system was used. Samples were placed into a stainless-steel cup, added with $5 \mu \mathrm{L}$ of TMAH solution and inserted into the micro-furnace. The pyrolysis temperature was set at $600^{\circ} \mathrm{C}$ for $0.2 \mathrm{~min}$, the interface temperature was $300{ }^{\circ} \mathrm{C}$ and the temperature of the GC injector was kept at $280{ }^{\circ} \mathrm{C}$. The GC was a $6890 \mathrm{~N}$ Network GC System (Agilent Technologies, Wilmington, DE, USA) gas chromatograph with a methylphenyl-polysiloxane cross-linked 5\% phenyl methyl silicone ( $30 \mathrm{~m}, 0.25 \mathrm{~mm}$ i.d., $0.25 \mu \mathrm{m}$ film thickness) capillary column. The carrier gas was helium $(1.0 \mathrm{~mL} / \mathrm{min})$ and split ratio was $1 / 20$ of the total flow. The mass spectrometer coupled to the GC apparatus was a 5973 Network Mass Selective Detector (Agilent Technologies, Wilmington, DE, USA). Mass spectra were recorded under electron impact at $70 \mathrm{eV}$, scan range $40-600 \mathrm{~m} / \mathrm{z}$. The interface was kept at $280^{\circ} \mathrm{C}$, ion source at $230^{\circ} \mathrm{C}$ and quadrupole mass analyzer at $150^{\circ} \mathrm{C}$. All instruments were controlled by Enhanced Chem Station (ver. 9.00.00.38) software. The mass spectra assignment was done with the Wiley 138 and NIST1992 libraries and by comparison with literature data.

\section{Results}

The efficacy of chemical and laser cleaning methods was assessed by a multi-analytical approach. In this section data and results of the cleaning tests and of the analytical characterization of the cleaned surfaces are described while more general comments and considerations on the usefulness and limits of the various cleaning methods, as well as the analytical approach used in this study, will be reported in the following discussion section.

\subsection{Mock-up A: Green Styrene-Acrylic Paint + Red Styrene-Acrylic Paint}

The cleaning purpose in this stratigraphy was the removal of the red styrene-acrylic paint from the green styrene-acrylic one. As for all specimens, the protective coating was applied over the first layer of paint (the green one), only on the right half portion.

The formulations tested on this mock-up were agar gel with ethanol (formulation II) for $5 \mathrm{~min}$ and the Nanorestore Gel ${ }^{\circledR}$ Extradry with a solution of Polar Coating $S^{\circledR}$ in 9\% Ethyl acetate (formulation III) applied for $1 \mathrm{~h}$.

Formulation II, followed by a final rinse with the same solution embedded into the gel, was found to be weakly selective. Nevertheless, some traces of the red styrene-acrylic paint were clearly observed both on the unprotected and protected area (A_II_np and A_II_wp respectively; Figure 2). In particular, on A_II_np, also the green layer was removed revealing the support in some points. Instead, A_II_wp seemed better preserved even if colorimetric measurements highlighted a colour difference higher than the human eye threshold (i.e., $\Delta E>3$ ) $[34,35]$ both with or without protective coating (Table 3).

Table 3. Colorimetric measurements on unprotected and protected areas after the cleaning procedure II and III.

\begin{tabular}{ccccc}
\hline Area & $\boldsymbol{\Delta} \boldsymbol{L}^{*}$ & $\boldsymbol{\Delta} \boldsymbol{a}^{*}$ & $\boldsymbol{\Delta} \boldsymbol{b}^{*}$ & $\boldsymbol{\Delta} \boldsymbol{E}$ \\
\hline A_II_np & -1.90 & 4.62 & 0.79 & 5.06 \\
A_II_wp & 0.91 & 2.97 & -2.92 & 4.26 \\
A_III_np & -0.78 & 5.51 & 1.30 & 5.72 \\
A_III_wp & -1.05 & 3.63 & -2.18 & 4.36 \\
\hline
\end{tabular}




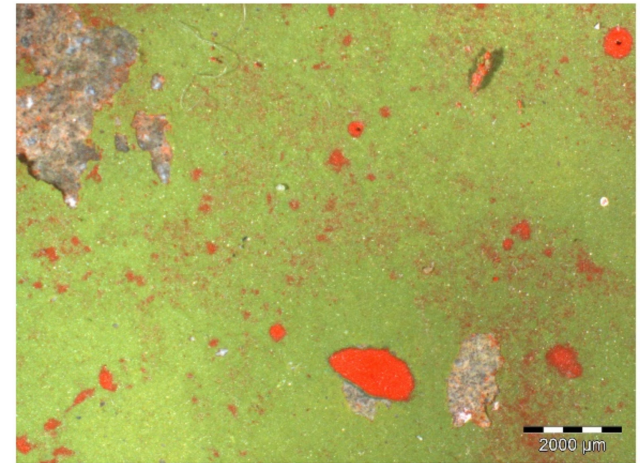

A_II_np

(a)

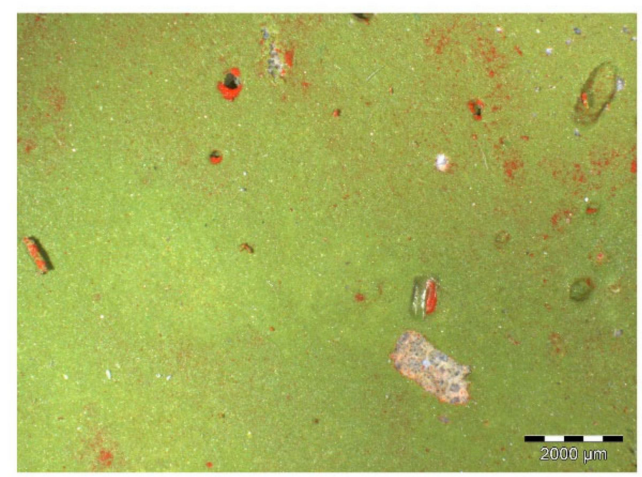

A_II_wp

(c)

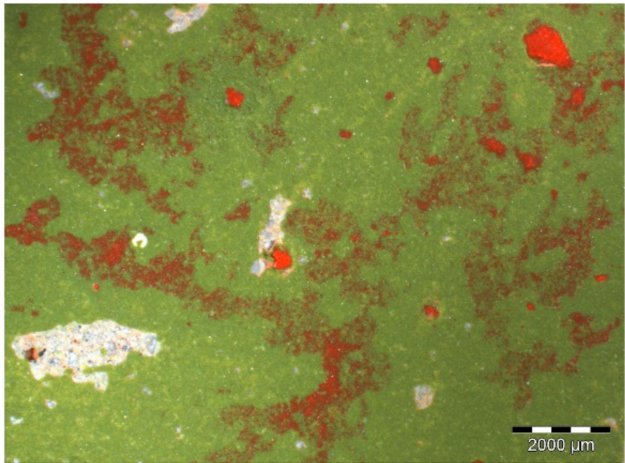

A_III_np

(b)

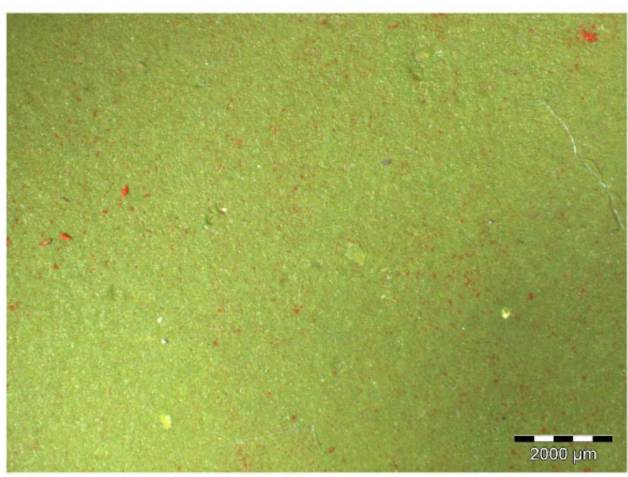

A_III_wp

(d)

Figure 2. Optical images at $10 \times$ of mock-up A treated with agar gel with ethanol $(\mathbf{a}, \mathbf{c})$ and with Nanorestore Gel ${ }^{\circledR}$ with a solution of Polar Coating $S^{\circledR}$ in $9 \%$ Ethyl acetate for $1 \mathrm{~h}(\mathbf{b}, \mathbf{d})$ on both unprotected (np) and protected (wp) areas. (a) A_II_np; (b) A_III_np; (c) A_II_wp; (d) A_III_wp.

After the 1-h application of Nanorestore Gel ${ }^{\circledR}$, followed by a final water rinse, the acrylic red paint showed a tendency to swell, especially in the protected area, as a consequence the cleaning test appeared tricky on the unprotected area (A_III_np): indeed, the cleaning was not fully effective and numerous residues of red paint were visible; on the other hand, as already observed with formulation II, sometimes the lower paint was removed and the support exposed (Figure 2).

On the contrary, the Nanorestore $\mathrm{Gel}^{\circledR}$ allowed to achieve acceptable results on the protected surface (A_III_wp) without any evident damage on the green layer underneath. However, signals related to the red pigment of the styrene-acrylic paint (at 1652, 1592, 1567, 1311, 942, and $713 \mathrm{~cm}^{-1}$ ) and to the protective coating (at 1258, 1089, and $797 \mathrm{~cm}^{-1}$ ) were identified by ATR-FTIR analyses on A_III_wp. Many of these signals were of low intensity, but of certain attribution by comparison with the spectrum of the reference styrene-acrylic paint. This finding is consistent with data reported in Table 3, showing that the color difference induced by the cleaning treatments was over the human eye threshold both for A_III_np and A_III_wp.

Laser cleaning was performed with a frequency range from 3 to $8 \mathrm{~Hz}$ in dry and wet condition and in some cases with the help of a gentle mechanical action with a brush. At a first glance, dry condition with lower frequency of $3 \mathrm{~Hz}$ and fluence approximately $0.75 \mathrm{~J} / \mathrm{cm}^{2}$ (LQS-I) was not effective in paint removal, both on the unprotected and protected area (A_IV_np_dry and A_IV_wp_dry respectively; Figure 3). Increasing the frequency up to $6 \mathrm{~Hz}$ with fluence approximately $1.35 \mathrm{~J} / \mathrm{cm}^{2}$ (LQS-I), the upper paint layer on A_IV_np_dry showed the tendency to swell: red flakes could be removed by mechanical action, but traces of green paint were visible on the back side. A similar situation occurred on 
A_IV_wp_dry, where the green paint layer underneath appeared etherogeneous and fewer green remains were observed on the back of the removed red flakes. The same fluence (i.e., approximatly $1.35 \mathrm{~J} / \mathrm{cm}^{2}$ ) with $8 \mathrm{~Hz}$ frequency was tested on the wet surface, covered by a Melynex ${ }^{\circledR}$ polyester film to slow down water evaporation. Samples A_IV_np_wet and A_IV_wp_wet exhibited a partial and uneven cleaning, but increasing the fluence up to approximately $2.60 \mathrm{~J} / \mathrm{cm}^{2}$ (LQS-I) the laser cleaning on the protected area showed slighly better results.

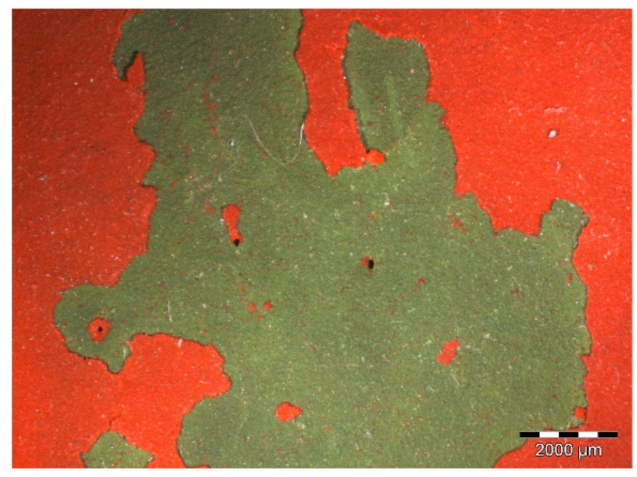

A_IV_np_dry

(a)

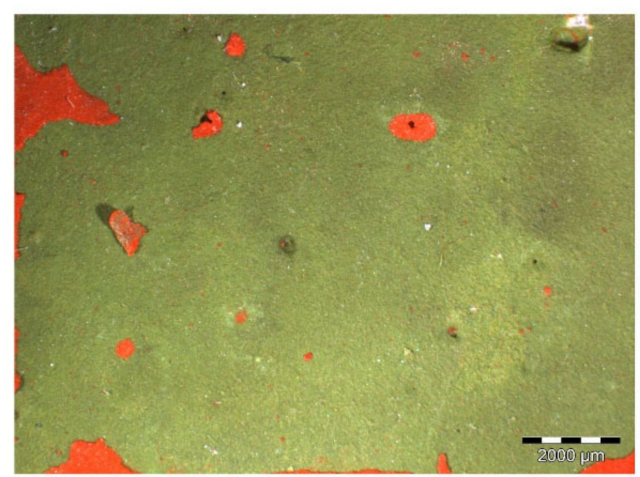

A_IV_wp_dry

(c)

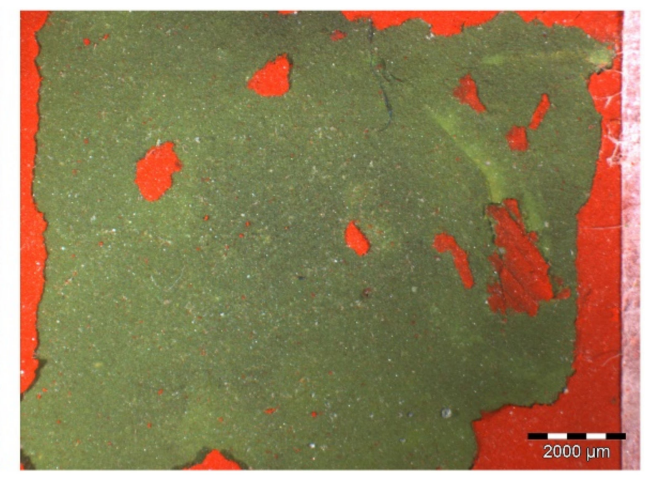

A_IV_np_wet

(b)

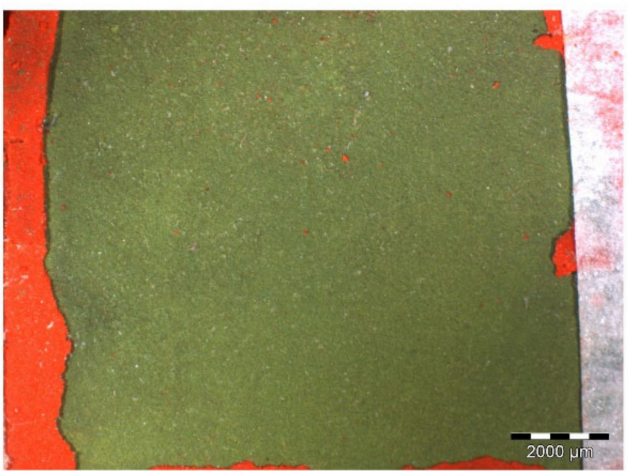

A_IV_wp_wet

(d)

Figure 3. Optical images at $10 \times$ of mock-up A treated with laser at $6 \mathrm{~Hz}$ frequency and fluence of approximately $1.35 \mathrm{~J} / \mathrm{cm}^{2}(\mathbf{a}, \mathbf{c})$ and at $6 \mathrm{~Hz}$ frequency and fluence of approximately $2.60 \mathrm{~J} / \mathrm{cm}^{2}(\mathbf{b}, \mathbf{d})$ both on unprotected (np) and protected (wp) area. (a) A_IV_np_dry; (b) A_IV_np_wet; (c) A_IV_wp_dry; (d) A_IV_wp_wet.

Finally, a further adjustment of laser parameters (fluence of approximately $2.60 \mathrm{~J} / \mathrm{cm}^{2}$ with $6 \mathrm{~Hz}$ frequency; LQS-II mode) allowed to achieve very interesting cleaning results: also in this case, the red paint layer swells due to the thermal confinement of the laser radiation. The outer layer is not directly ablated, but partially detached and thus easily peeled away by brush (Figure 3). Moreover, the red flakes removed from A_IV_np_wet showed few traces of green paint, which are not visible at all in the case of A_IV_wp_wet.

According to FTIR analyses, operating with a fluence of approximately $2.60 \mathrm{~J} / \mathrm{cm}^{2}$ and $6 \mathrm{~Hz}$ frequency (LQS-II) on the wet surface, laser cleaning was able to completely remove the red paint only on the protected area, while operating with a fluence of approximately $1.30 \mathrm{~J} / \mathrm{cm}^{2}$ and a frequency of $8 \mathrm{~Hz}$ (LQS-I) on a wet surface, the red paint was rather well removed regardless of the presence or absence of the protective coating. In these cases, the thermal reduction of the laser radiation thanks to the wetting agent and the protective coating, when present, also allows to better preserve the green paint. In general, the use of a protective coating is preferable because it always allows a better removal 
of the unwanted red paint, while if absent traces of red paint are still found over the green background both in wet and dry condition. Figure 4 shows the FTIR spectra recorded on sample A_IV_np_wet and A_IV_wp_wet, treated with laser at $6 \mathrm{~Hz}$ and fluence of approximately $2.60 \mathrm{~J} / \mathrm{cm}^{2}$ (LQS-II), and the spectrum of the reference green styrene-acrylic paint for comparison. The most intense peaks are attributed to vibrational modes of $\mathrm{CaCO}_{3}$, at 1408,873 and $712 \mathrm{~cm}^{-1}$, and talc, at 3675, 1014 and $670 \mathrm{~cm}^{-1}$. Characteristic absorptions of the styrene-acrylic binder are the $\mathrm{C}-\mathrm{H}$ and $=\mathrm{CH}$ stretching in the range $2800-3100 \mathrm{~cm}^{-1}$, the $\mathrm{C}=\mathrm{C}$ stretching at $1600 \mathrm{~cm}^{-1}$ and the $=\mathrm{C}-\mathrm{H}$ out-of-plane deformations at 757 and $698 \mathrm{~cm}^{-1}$, due to the styrene units, and the $\mathrm{C}=\mathrm{O}$ and $\mathrm{C}-\mathrm{O}$ stretching of acrylate units at 1728 and $1158 \mathrm{~cm}^{-1}$ respectively. In particular, the spectrum of sample A_IV_np_wet also shows some specific absorptions of the red pigment of the styrene-acrylic paint at 1655, 1571, 1312, 772 and $754 \mathrm{~cm}^{-1}$. These signals are absent in the spectrum collected on A_IV_wp_wet that instead shows two absorption bands of the protective coating at 1260 and $1090 \mathrm{~cm}^{-1}$.

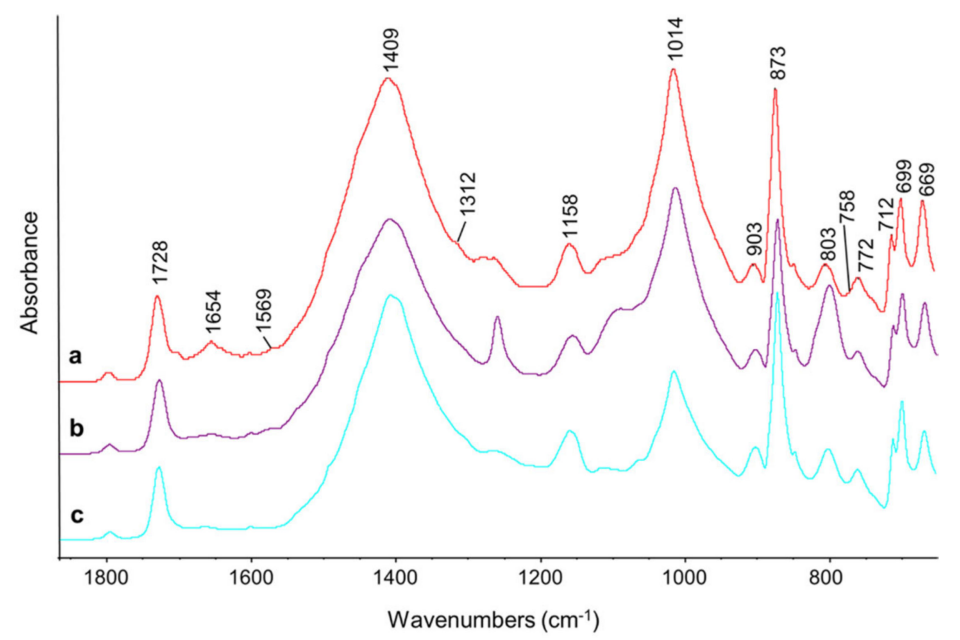

Figure 4. FTIR spectra of unprotected (np) and protected (wp) areas of mock-up A treated with laser $\left(6 \mathrm{~Hz}\right.$, fluence of approximately $2.60 \mathrm{~J} / \mathrm{cm}^{2}$, on wet surface). Labels in spectrum a show the absorptions due to the red styrene-acrylic residues. (a) A_IV_np_wet; (b) A_IV_wp_wet; (c) reference green styrene-acrylic paint.

\subsection{Mock-up B: Green Alkyd Spray Paint + Red Alkyd Spray Paint}

The purpose of the cleaning in this stratigraphy was the removal of the red alkyd spray paint from the green alkyd.

Removing selectively one painting layer characterized by the same chemical composition of the painting layer underneath is a very difficult situation in terms of possible cleaning achievements because of the same solubility range of the two painting layers. The removal of the red alkyd paint from the green alkyd one was faced both with a free solvent and a thickener: propylene carbonate (formulation I) applied with a micro-swab and Nanorestore Gel ${ }^{\circledR}$ Extradry with a solution of Polar Coating $S^{\circledR}$ in 9\% Ethyl acetate for $1 \mathrm{~h}$ (formulation III), rinsed with water.

A selective removal of the outermost alkyd layer is to be considered impossible with both formulations. Unfortunately, the cleaning was found to be non-selective because also the green alkyd layer underneath was removed. Figure 5 shows the complete removal of the whole paint stratigraphy with no difference between the protected and the unprotected area. Only few paint remains are visible in the porosity of the surface. Therefore, any further analytical analyses were considered useless. 


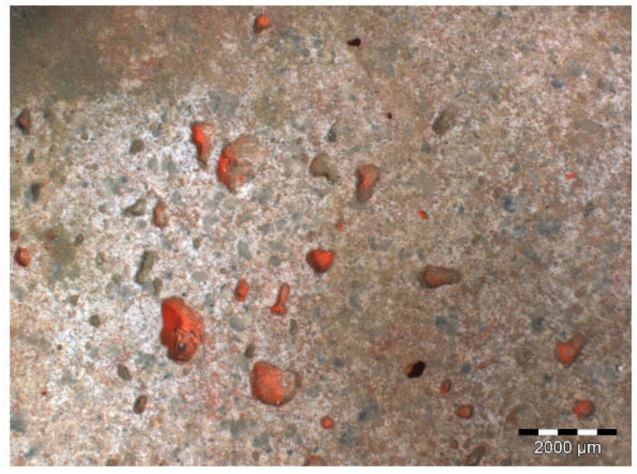

B_I_np

(a)

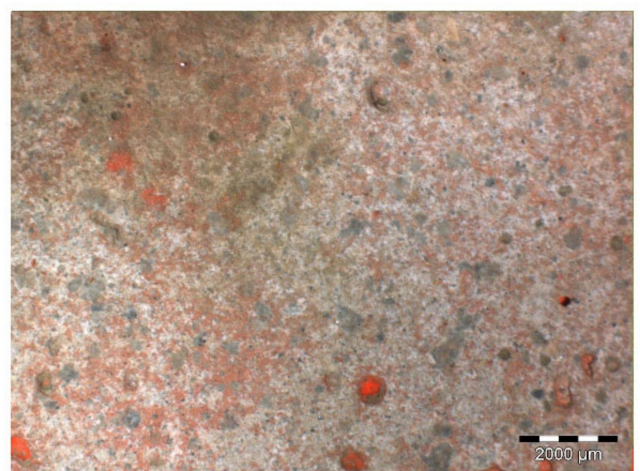

B_I_wp

(c)

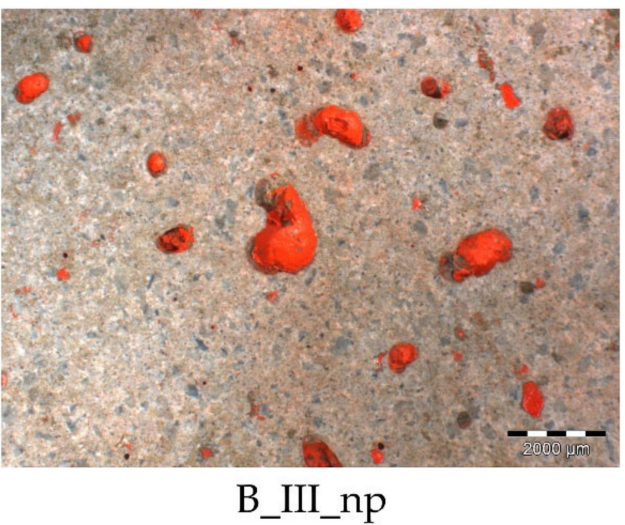

(b)

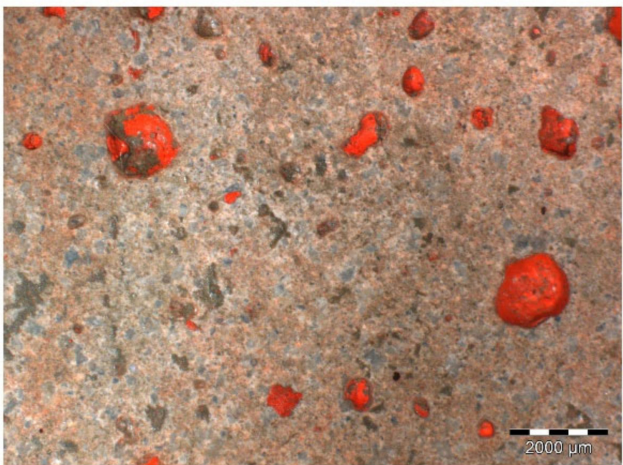

B_III_wp

(d)

Figure 5. Optical images at $10 \times$ of mock-up A treated with propylene carbonate $(\mathbf{a}, \mathbf{c})$ and Nanorestore $\mathrm{Gel}^{\circledR}$ for $1 \mathrm{~h}(\mathbf{b}, \mathbf{d})$ on both the unprotected (np) and the protected (wp) area. (a) B_I_np; (b) B_III_np; (c) B_I_wp; (d) B_III_wp.

Laser cleaning also did not lead to satisfactory results. Both protected and unprotected areas were tested in dry and wet condition using the same parameters (frequency and fluence) and in some cases with a gentle mechanical action with a brush. Moreover, to slow down water evaporation a Melynex ${ }^{\circledR}$ polyester film was used to cover the treated area.

On the unprotected portion of the specimen (B_IV_np_dry), the use of laser with fluence around $0.78 \mathrm{~J} / \mathrm{cm}^{2}$ (LQS-I) on the dry surface proved to be only slightly selective in removing the outer red alkyd spray paint layer from the green one underneath. The damage threshold was easily reached exposing the support, and this occurred even to a greater extent in the protected portion (B_IV_wp_dry). In order to enhance the laser efficacy, the same parameters were used on the wet surface. However, also in this case selectivity was not achieved either on B_IV_np_wet or B_IV_wp_wet. Likewise, even rising the fluence at approximately $1.35 \mathrm{~J} / \mathrm{cm}^{2}$ (LQS-I) and operating in wet condition, no improvements were recorded and the damage threshold was quickly overcome (Figure 6). On B_IV_np_wet, a first step of cleaning seemed to remove gently the outer layer, while with further steps the selectivity was easily lost. Moreover, the protective coating seemed to be strongly adherent to the green paint layer enhancing the removal of the entire paint stratigraphy during the laser treatment.

As for the chemical cleaning tests, results were not considered satisfactory and no other analysis was performed. 


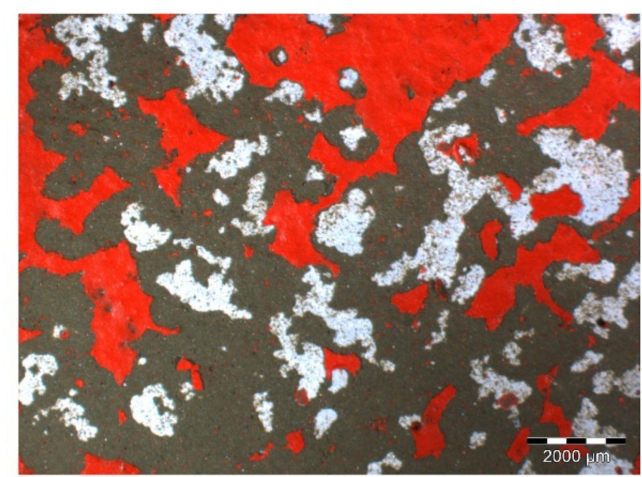

B_IV_np_dry

(a)

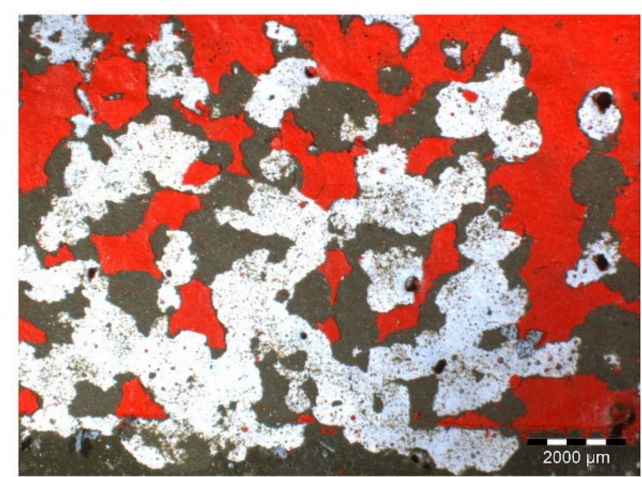

B_IV_wp_dry

(c)

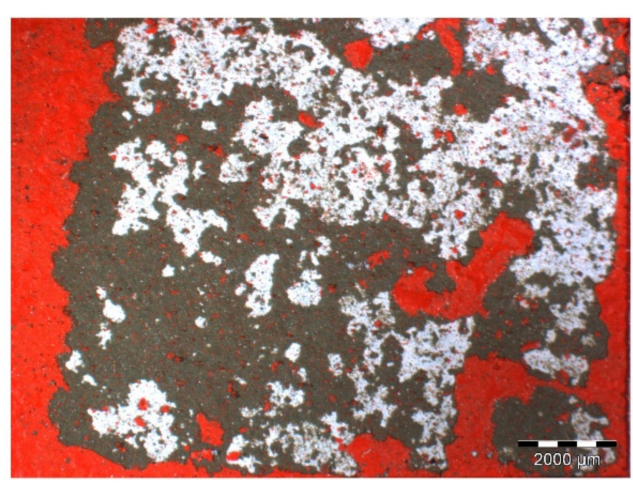

B_IV_np_wet

(b)

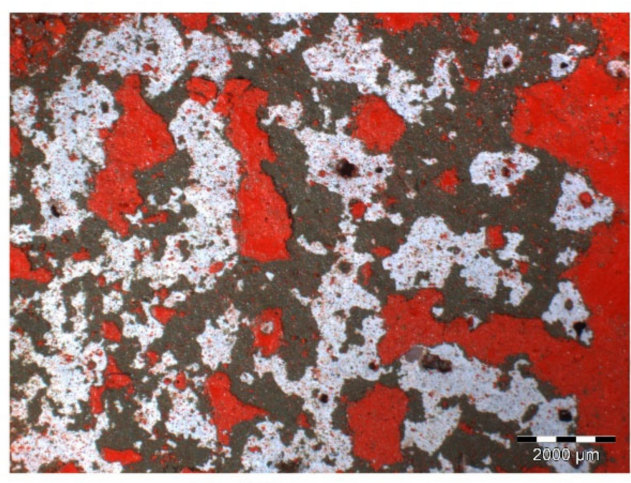

B_IV_wp_wet

(d)

Figure 6. Optical images at $10 \times$ of mock-up Btreated with laser cleaning with fluence approximately $0.78 \mathrm{~J} / \mathrm{cm}^{2}$ on dry surface (a,c) and with fluence approximately $1.35 \mathrm{~J} / \mathrm{cm}^{2}$ on wet surface $(\mathbf{b}, \mathbf{d})$, both on unprotected (np) and protected (wp) areas. (a) B_IV_np_dry; (b) B_IV_wet; (c) B_IV_wp_dry; (d) B_IV_wp_wet.

\subsection{Mock-up C: Green Alkyd Spray Paint + Red Styrene-Acrylic Paint}

The cleaning formulations tested on this stratigraphy were the same of the previous one, i.e., formulation I and formulation III, in this case with a time of application of $30 \mathrm{~min}$, then raised to $1 \mathrm{~h}$.

Free propylene carbonate, applied with a micro-swab gently rolled on the surface, allowed to reach a good removal of the red styrene-acrylic paint without a strong mechanical action, both on unprotected (C_I_np) and protected (C_I_wp) areas. Moreover, if associated with a soft mechanical action, traces of the unwanted paint could be easily removed without affecting the painting layer underneath, as reported in Figure 7. Nevertheless, the damage threshold seemed to be easily reached insisting in rolling the swab on the surface, showing that the increase of the amount of solvent interacting with the acrylic paint caused the solubilization of the paint underneath. Indeed, a thinning and the loss of glossy finishing was already observed after just a brief cleaning treatment. Colorimetric measurements reported in Table 4 are consistent with the macroscopic observations, showing $\Delta E$ values around 1 or lower. In particular, the presence of a protective coating allowed to preserve the original color of the green alkyd background during the cleaning, resulting in a low $\Delta E$ value. 


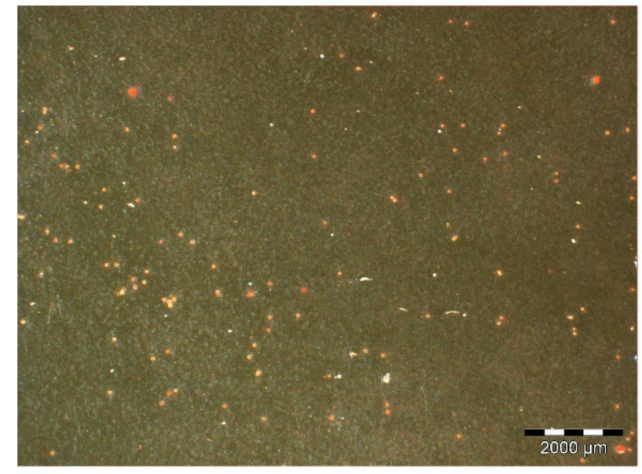

C_I_np

(a)

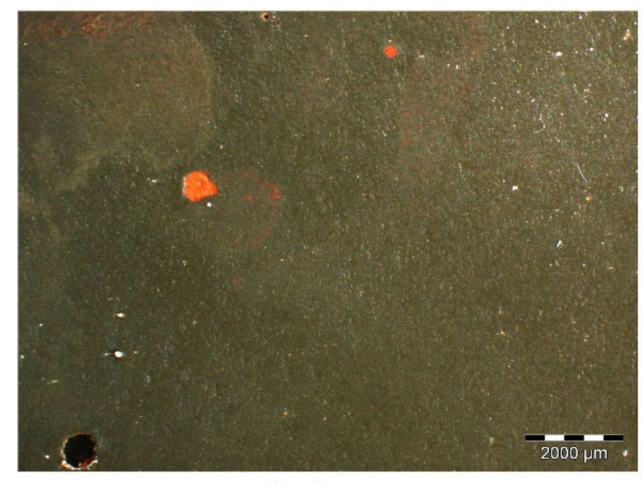

C_I_wp

(c)

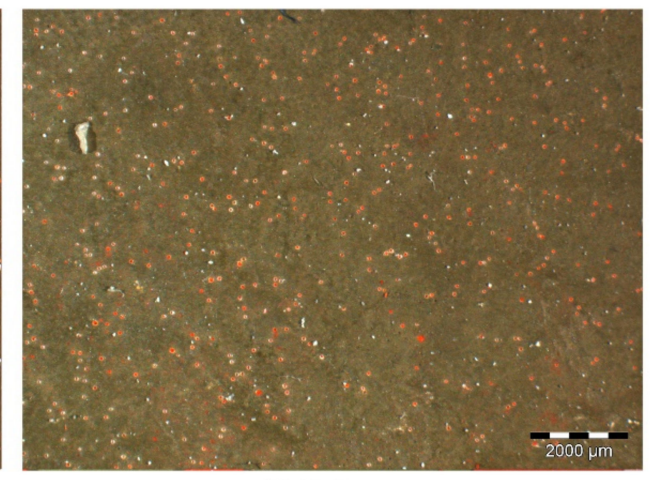

C_III_np

(b)

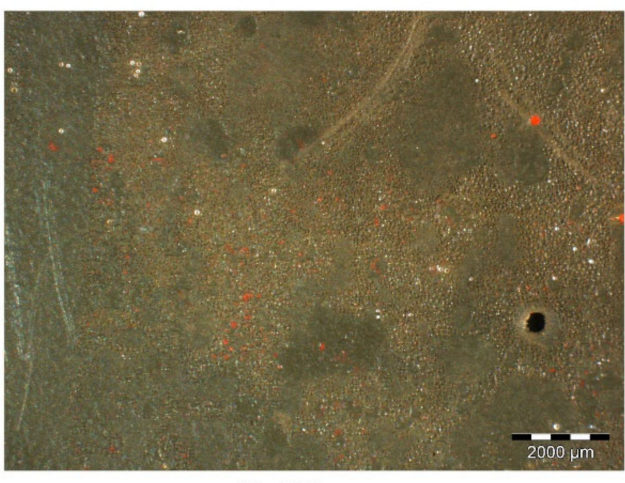

C_III_wp

(d)

Figure 7. Optical images at $10 \times$ of mock-up $C$ treated with propylene carbonate $(\mathbf{a}, \mathbf{c})$ on both unprotected and protected area, and with Nanorestore $\mathrm{Gel}^{\circledR}(\mathbf{b}, \mathrm{d})$ applied for $1 \mathrm{~h}$ and $30 \mathrm{~min}$ on the unprotected (np) and the protected (wp) area respectively. (a) C_I_np; (b) C_III_np; (c) C_I_wp; (d) C_III_wp.

Table 4. Colorimetric measurements on unprotected and protected areas after the cleaning procedure I and III.

\begin{tabular}{ccccc}
\hline Area & $\boldsymbol{\Delta} \boldsymbol{L}^{*}$ & $\boldsymbol{\Delta} \boldsymbol{a}^{*}$ & $\boldsymbol{\Delta} \boldsymbol{b}^{*}$ & $\boldsymbol{\Delta} \boldsymbol{E}$ \\
\hline C_I_np & 0.04 & 0.50 & 1.02 & 1.13 \\
C_I_wp & -0.56 & 0.34 & 0.29 & 0.72 \\
C_III_np (1 h) & 8.18 & 2.92 & 4.16 & 9.62 \\
C_III_wp (30') & 1.84 & 1.14 & 2.05 & 2.98 \\
\hline
\end{tabular}

The complete removal of the red paint was also confirmed by FTIR and Py-GC/MS analyses. Figure 8 shows the infrared spectrum of area C_I_np, compared with those of the red styrene-acrylic reference and the green alkyd paint reference. The spectrum of C_I_np does not show the characteristic absorptions of the styrene-acrylic paint at 3100, 1600, 1408, 1158, 873, 757, 698 and $712 \mathrm{~cm}^{-1}$, but only those of the alkyd paint underneath. The same result was also obtained for C_I_wp. The FTIR spectrum collected on area C_I_wp is shown in Figure 9, together with the spectrum of the protective coating applied on the green paint, and again it does not show peaks that can be uniquely assigned to the red styrene-acrylic paint or to the siloxane coating. Then, also Py-GC/MS analyses were carried out on both cleaning areas in order to check whether, by using an analytical technique more sensitive than FTIR, it was possible to identify the residues either of the red styrene-acrylic paint or the protective coating. However, also in this case the corresponding signals were not detected. 


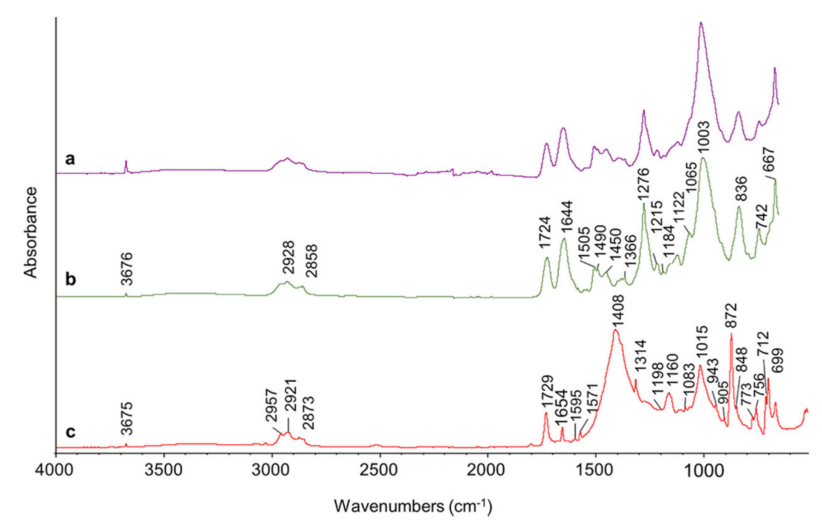

Figure 8. FTIR spectrum of the unprotected $(\mathrm{np})$ area of mock-up $\mathrm{C}$ treated with propylene carbonate, compared with spectra of reference sprays. (a) C_I_np; (b) reference green alkyd spray; (c) reference red alkyd spray.

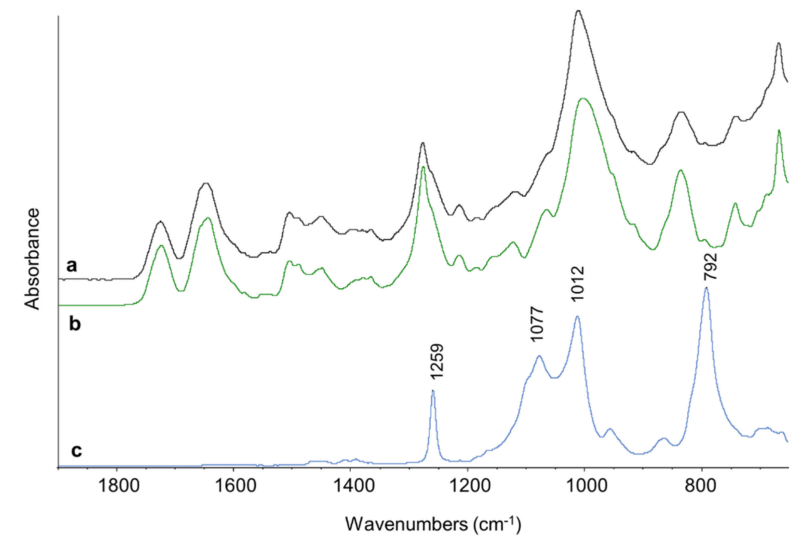

Figure 9. FTIR spectrum of the protected (wp) area of mock-up C treated with propylene carbonate, compared with spectra of the reference green alkyd spray and of the protective coating. (a) C_I_wp; (b) reference green alkyd spray; (c) protective coating.

As for the use of free propylene carbonate, also the application of Nanorestore Gel ${ }^{\circledR}$ for 30 min gave a better result on the protected area (C_III_wp) than on the unprotected one (C_III_np). Indeed, the gel was more efficient where the protective coating was present, while a longer application time (up to $1 \mathrm{~h}$ ) was required if the protective coating was absent (Figure 7). Moreover, the cleaning result appeared more homogeneous if the treatment was followed by a final rinse with the same liquid phase that swells the gel instead of water. However, colorimetric measurements clearly exhibited a visible colour change of the surfaces, either for $30 \mathrm{~min}$ or for $1 \mathrm{~h}$ (Table 4 ). The $\Delta E$ value significantly exceeds the human eye threshold in C_III_np, whereas it is lower in C_III_wp, highlighting a positive effect of the protective coating and possibly of the shorter application time.

FTIR and Py-GC/MS analyses confirmed the successful removal of the styrene-acrylic paint especially on area C_III_wp, where traces of the siloxane protective coatings were found by Py-GC/MS. In particular, three weak signals related to pyrolysis fragments of the protective coating have been identified by extracting from the TIC curve the profiles of characteristic fragment ions (i.e., 207, 281, $355 \mathrm{~m} / \mathrm{z}$ ) of cyclosiloxanes. The presence of traces of the protective coating in area C_III_wp indicates that the cleaning was more selective and left the alkyd layer unaltered, as confirmed by the $\Delta E$ value, which is much lower than on the unprotected portion.

As for the other laser cleaning, many preliminary tests were performed on the dedicated specimen to identify suitable working parameters. A range of frequencies from 5 to $10 \mathrm{~Hz}$ was tested on dry and wet surfaces. On dry surfaces, the thinning of the red styrene-acrylic paint layer caused a slight chromatic alteration. Wetting the surface and using a higher frequency $(8 \mathrm{~Hz})$, better results in terms of 
paint removal were obtained, but with some evident laser spot marks on the green surfaces underneath. It is worth to notice that the use of laser on wet surfaces with lower frequency values and the help of the mechanical action of a trimmed brush provided a partial removal of the upper paint layer; however, the removed red scales had some traces of green paint on their back sides, thus showing a limited selectivity of the cleaning procedure. Further set-ups were tested to ease the cleaning procedure: a Melynex ${ }^{\circledR}$ polyester film was applied on the surface to slow down water evaporation. This seemed to reduce the risk of chromatic alteration on the lower green paint layer, making the use of laser more controllable. Indeed, a slightly good result in cleaning efficacy was reached on the unprotected area when using this particular set-up and with a fluence of approximately $4.14 \mathrm{~J} / \mathrm{cm}^{2}$ (LQS-I) and frequency $5 \mathrm{~Hz}$, however a consistent mechanical action was necessary to reach a satisfactory paint removal. Thus, the same fluence associated with a higher frequency $(7 \mathrm{~Hz})$ was tested but again poor results were obtained on the unprotected area (C_IV_np_wet), while a better removal of the red paint was achieved on the protected one (C_IV_wp_wet), as shown in Figure 10.

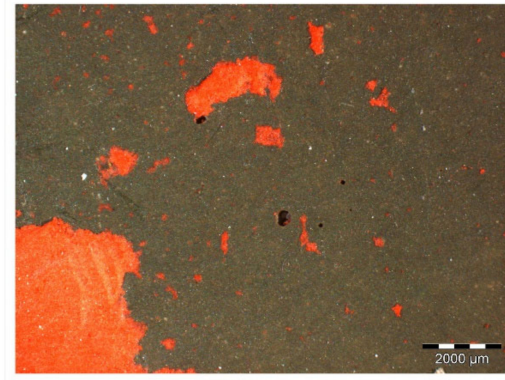

C_IV_np_wet

(a)

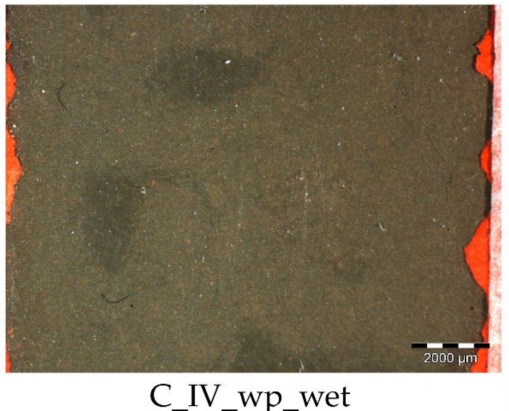

(b)

Figure 10. Optical images at $10 \times$ of mock-up $C$ treated with laser at $7 \mathrm{~Hz}$ frequency and fluence of approximately $4.14 \mathrm{~J} / \mathrm{cm}^{2}$ on unprotected (np) and protected (wp) area. (a) C_IV_np_wet; (b) C_IV_wp_wet.

However, traces of the red styrene-acrylic paint were detected by FTIR on both cleaning areas. Figure 11 shows as an example the FTIR spectrum of C_IV_wp_wet after the laser cleaning $(7 \mathrm{~Hz}$, $4.14 \mathrm{~J} / \mathrm{cm}^{2}$ ). Most of the peaks can be assigned to the green alkyd spray paint, but some absorptions characteristic of the red paint are still visible: the $\mathrm{CO}_{3}^{2-}$ asymmetric stretching at $1398 \mathrm{~cm}^{-1}$ and the $\mathrm{CO}_{3}^{2-}$ out-of-plane bending at $871 \mathrm{~cm}^{-1}$, due the $\mathrm{CaCO}_{3}$ present in the red paint.

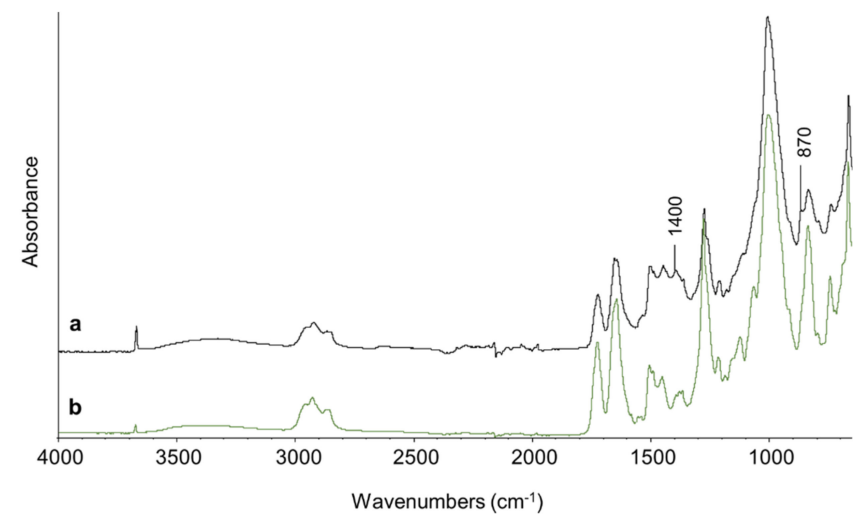

Figure 11. FTIR spectrum of the protected (wp) area of mock-up C treated with propylene carbonate, compared with the reference green alkyd spray. Labels in spectrum a show the absorptions due to the red styrene-acrylic residues. (a) C_IV_wp_wet; (b) reference green alkyd spray. 


\subsection{Mock-up D: Red Styrene-Acrylic Paint + Green Alkyd Spray Paint}

The paints used in these mock-ups were the same as mock-up C, but with a reversed stratigraphy. The purpose was to study the efficacy of the same cleaning methods (formulations I and III) when the order of the two paint layers was different, i.e., when a green alkyd spray paint has to be removed from a red styrene-acrylic paint.

As reported in Figure 12, the propylene carbonate applied with a micro-swab solubilized efficiently the green alkyd layer, without removing the underneath red styrene-acrylic one, both in the unprotected and the protected area (D_I_np and D_I_wp). However, few stains were visible in the D_I_np area. This drawback was confirmed by the colorimetric measurements. In fact, the colour change value was acceptable and lower than the threshold of the human eye in D_I_wp, while it significantly exceeds this value in D_I_np (Table 5).

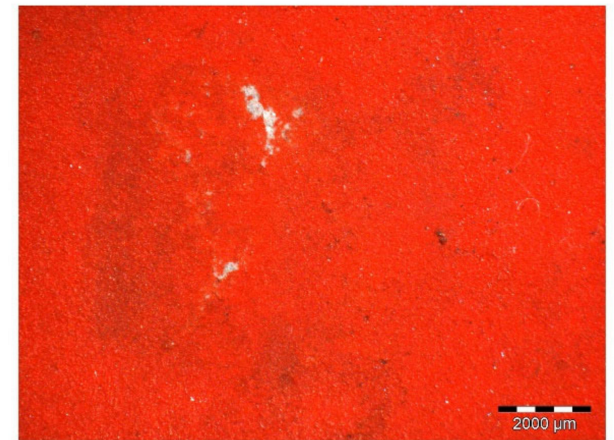

D_I_np

(a)

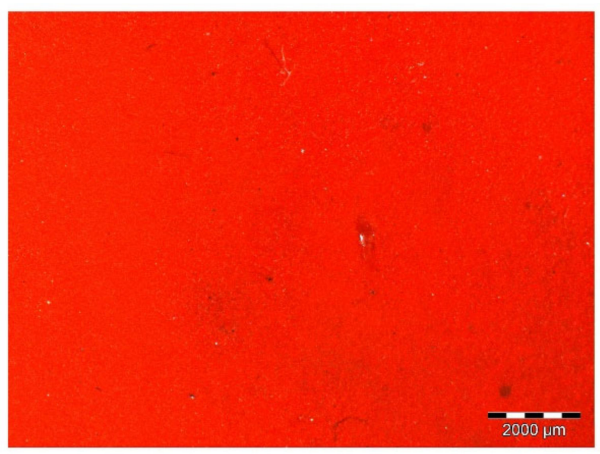

D_I_wp

(c)

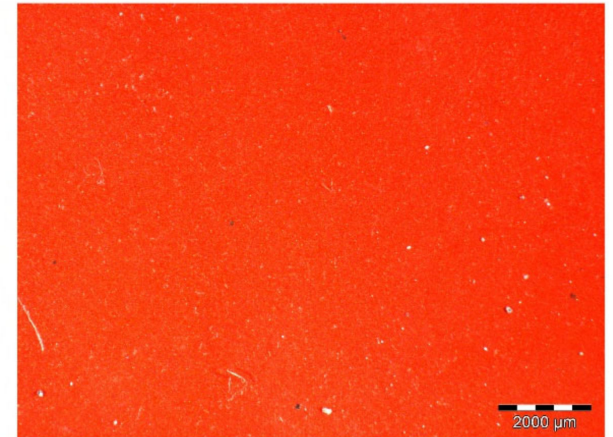

D_III_np

(b)

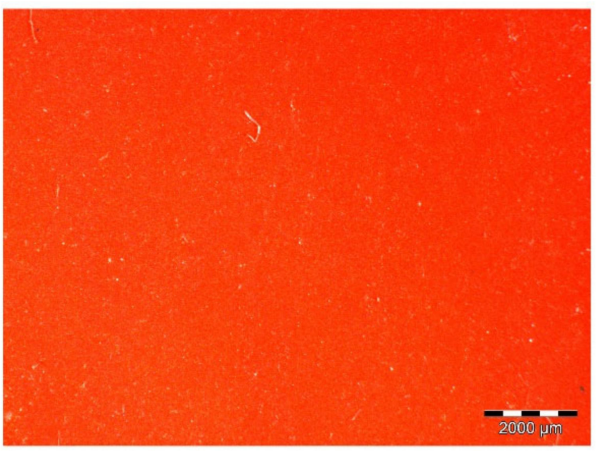

D_III_wp

(d)

Figure 12. Optical images at $10 \times$ magnification of mock-up $D$ treated with propylene carbonate solvent $(\mathbf{a}, \mathbf{c})$ and Nanorestore $\mathrm{Gel}^{\circledR}$ gel for $30 \mathrm{~min}(\mathbf{b}, \mathbf{d})$ on the unprotected and protected area. (a) D_I_np; (b) D_III_np; (c) D_I_wp; (d) D_III_wp.

Table 5. Colorimetric measurements on unprotected and protected areas, respectively after application of propylene carbonate and of Nanorestore $\mathrm{Gel}^{\circledR}$ (for $30 \mathrm{~min}$ ).

\begin{tabular}{ccccc}
\hline Sample & $\boldsymbol{\Delta} \boldsymbol{L}^{*}$ & $\boldsymbol{\Delta} \boldsymbol{a}^{*}$ & $\boldsymbol{\Delta} \boldsymbol{b}^{*}$ & $\boldsymbol{\Delta} \boldsymbol{E}$ \\
\hline D_I_np & -0.71 & -4.21 & -2.60 & 4.99 \\
D_I_wp & -1.65 & -0.30 & 1.02 & 1.96 \\
D_III_np (30') & -0.91 & -5.72 & -3.17 & 6.60 \\
D_III_wp (30') & -1.10 & -2.56 & -0.60 & 2.85 \\
\hline
\end{tabular}

On the other hand, Nanorestore Gel ${ }^{\circledR}$ Extradry applied for 30 min allowed a quite controllable cleaning, that was even more efficient if associated with a final rinse by using Polar Coating $S$ instead 
of water. Therefore, the use of Nanorestore Gel ${ }^{\circledR}$ Extradry seemed a good option for a selective and efficient removal of the green alkyd spray paint, but, especially in the unprotected area (D_III_np), it resulted in localized tearing of the red styrene-acrylic layer (Figure 12). The colorimetric measurements confirmed this trend with a $\Delta E$ value moderately lower than the human eye threshold for D_III_wp and significantly higher for D_III_np.

The different samples analysed by ATR-FTIR after the cleaning provided very similar results: in none of the spectra it was possible to recognize the presence of the green alkyd paint, while in the areas D_I_wp and D_III_wp the characteristic peaks of the siloxane protective (at 1260, 1077, and $797 \mathrm{~cm}^{-1}$ ) were identified. However, it should be noted that the spectrum of the styrene-acrylic paints shows intense and wide absorptions due to the presence of calcite which overlap with many absorptions characteristic of the alkyd paint, while other absorptions due to talc or to some vibrational modes of the binder are common to both alkyd and acrylic paint, making it difficult to identify any traces of alkyd paint that were not removed.

On the other hand, the alkyd spray paint was detected by Py-GC/MS analyses in all cases, even in the area with the protective coating for which cleaning looked satisfactory to the naked eye and the $\Delta E$ value was lower than 3 . This is because Py-GC/MS with thermally assisted hydrolysis and methylation using (THM) is particularly sensitive to the presence of alkyds, giving pyrograms with intense peaks of the main components of alkyd resins, i.e., methylated fatty acids, acids and polyalcohols. As an example, in the pyrogram of D_I_np, reported in Figure 13, peaks 7, 8, 10, 13, 14 and 15 are markers of methylated pentaerythritol (i.e., 2,2-bis(hydroxymethyl)propane-1,3-diol), a typical polyol used in the synthesis of alkyd resins, peak 10 is a marker of phthalic anhydride, another typical monomer used in the synthesis of alkyds, and peaks 13, 14, and 15 are methylated fatty acids from the oil fraction of alkyd resins. Most of the other peaks (i.e., 1, 2, 3, 4, 5, 6, 9, 11, 12, and 16) can be assigned to pyrolysis fragments characteristic of the styrene-acrylic paint.

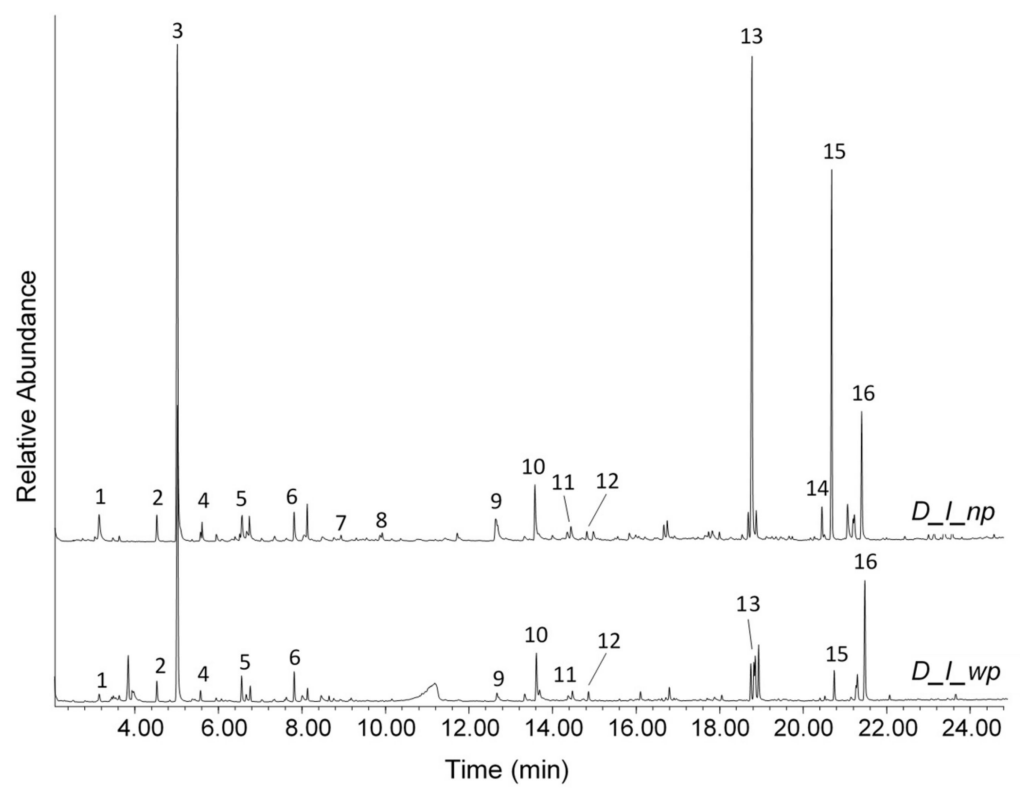

Figure 13. Py-GC/MS curves of samples D_I_np and D_I_wp showing characteristic markers of the lower styrene-acrylic paint layer and residues of the upper alkyd spray. Toluene (1); ethylbenzene (2); styrene (3); (1-methylethyl)-benzene (4); $\alpha$-methylstyrene (5); (1-methylenepropyl)-benzene (6); 1,3-dimethoxy-2,2-bis(methoxymethyl)-propane (7); 1,3-dimethoxy-2,2-bis(methoxymethyl)- propane (8); biphenyl (9); dimethyl phthalate (10); 1,1'-(1,2-ethanediyl)bis-benzene (11); 1,1'-(1-methyl-1,2ethanediyl)bis-benzene (12); hexadecenoic acid methyl ester (13); octadecenoic acid methyl ester (14); octadecanoic acid methyl ester (15); m-terphenyl (16). 
The pyrogram obtained from sample D_I_wp (Figure 13), which corresponds to the best cleaning result according to color measurements and microscope observation, still shows peaks related to the presence of the alkyd paint (n. 7, 10,13,14, and 15), although if compared to the styrene-acrylic markers their intensity is very low. This confirms that the presence of the siloxane coating and the use of Nanorestore Gel ${ }^{\circledR}$ Extradry applied for $30 \mathrm{~min}$ is the approach that allows to get the most selective and effective removal of an alkyd paint sprayed on top of an acrylic paint. In the pyrogram of D_I_wp very tiny peaks (not shown in Figure 13) of siloxane fragments due to the protective coatings were also detected.

Concerning the laser cleaning, the most suitable parameters tested for the previous specimens unfortunately did not fit exactly with the current one. Neither repeating steps with $7 \mathrm{~Hz}$ frequency and fluence of approximately $4.14 \mathrm{~J} / \mathrm{cm}^{2}$ (LQS-I), nor keeping the surface wet, the laser selectivity did not improve on the unprotected area (D_IV_np_wet). An equal result was recorded on the protected portion of the specimen (D_IV_wp_wet), where either a thinning or an uncomplete removal of the green alkyd layer was obtained (Figure 14). Reducing slightly the frequency, but with the same fluence and working on a dry surface, none improvement was achieved: the results were heterogeneous, and just a thinning of the green alkyd paint was obtained both on D_IV_np_dry and D_IV_wp_dry (Figure 14). Moreover, it is worth to notice that the protective coating seemed to be strongly adherent with the lower red styrene-acrylic paint, probably due to a partial solubilization during the stratigraphy preparation.

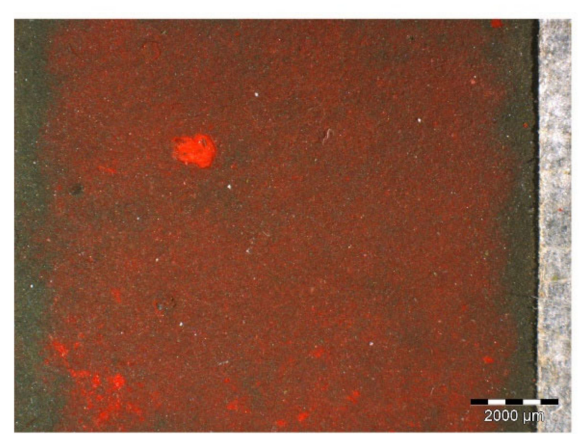

D_IV_np_wet

(a)

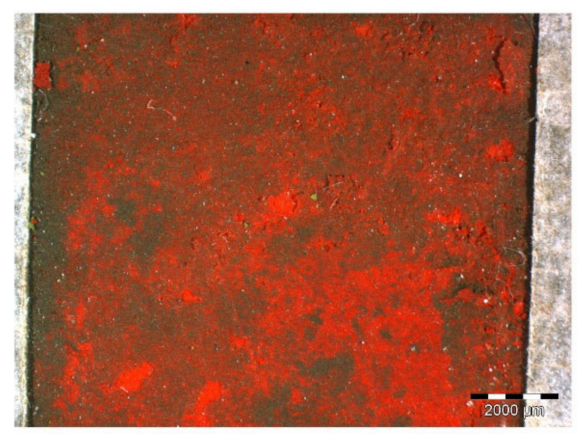

D_IV_wp_wet

(c)

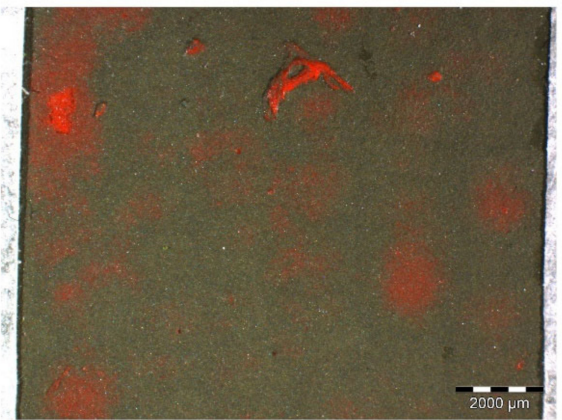

D_IV_np_dry

(b)

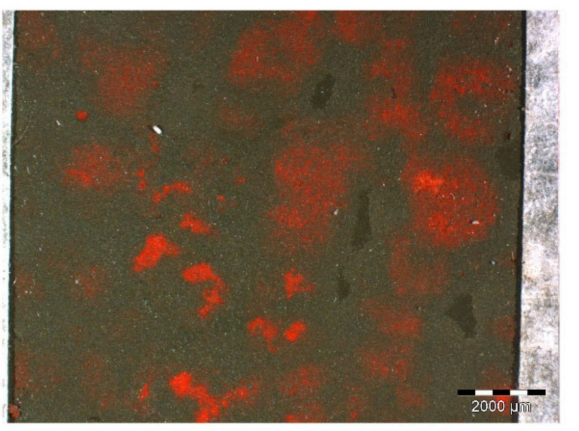

D_IV_wp_dry

(d)

Figure 14. Optical images at $10 \times$ magnification of mock-up $D$ treated with laser at $7 \mathrm{~Hz}$ frequency and fluence of $4.14 \mathrm{~J} / \mathrm{cm}^{2}$ on wet surfaces $(\mathbf{a}, \mathrm{c})$ and $6 \mathrm{~Hz}$ frequency and fluence of $4.14 \mathrm{~J} / \mathrm{cm}^{2}$ on dry surfaces (b,d), both on unprotected (np) and protected (wp) areas. (a) D_IV_np_wet; (b) D_IV_np_dry; (c) D_IV_wp_wet; (d) D_IV_wp_dry.

\section{Discussion}

Unfortunately, none of the cleaning methods tested proved to be fully satisfactory and effective, all having a significant impact on the cleaned surfaces. As expected, the most critical case consisted in 
the need of removing a paint layer deriving from an act of vandalism and characterized by the same chemical composition of the paint underneath (i.e., acrylic + acrylic; alkyd + alkyd).

This limit appeared clearly for sample B, where none of the cleaning methods tested gave acceptable results. The red alkyd paint firmly adhered to the already dried green layer, so much that the various approaches to selectively remove the outermost paint led either to a partial removal or, more often, to a removal of the two layers together (both the red one and the green underneath). Reasonably, this can be explained considering that the hardening process of an alkyd resin depends not only on the evaporation of the solvent, but mainly on the auto-oxidative cross-linking reaction which involves the fatty acid chains of the alkyd resins. These fatty acids contain bis-allylic hydrogen atoms $\left(=\mathrm{C}-\mathrm{CH}_{2}-\mathrm{CH}=\right)$, which have a relatively low $\mathrm{C}-\mathrm{H}$ bond and can easily be abstracted to start a radical chain reaction. The radicals formed in this way reacts very rapidly with molecular oxygen to form peroxy radicals that then abstract hydrogen from other resin molecules to form a hydroperoxide, propagating the radical reaction [36]. These radicals can then combine to form addition products with ether or peroxy cross-links. A certain degree of co-crosslinking at the interface between the two alkyd layers is conceivable, which would make it difficult to separate the upper layer from the lower one.

Even the laser, despite the tuning of several operative parameters, was not able to remove selectively the unwanted outer layer. Indeed, during the test, the conservator observed a strong cohesion both between the painting layer without the protective coating and between this latter and the outer painter layer.

A slightly better outcome was obtained from the cleaning tests carried out on sample A, on which fewer paint remains were either visible or detected by instrumental analyses. In this regard, it is worth noting that the diagnostic approach used in this study first involved careful observation of the samples with the naked eye or under the microscope, during and after the cleaning test. Only if no traces of the upper paint layer were found from this observation, FTIR analysis was performed and only when the residues of the removed paint were not detected by FTIR then Py-GC/MS was used, being a more sensitive technique than FTIR, although less accessible. In the case of the stratigraphy of type A, cleaning test performed with Nanorestore Gels ${ }^{\circledR}$ and laser on samples A_III_wp, A_IV_np_dry and A_IV_np_wet gave acceptable results at the naked eye, but signals of the upper red paint layer were clearly detected by FTIR. On the other hand, on samples A_IV_wp_dry and A_IV_wp_wet no residues of the upper paint layer were found, while signals of the protective coating were detected, even after the cleaning, which proves that in the case of laser cleaning the presence of a protective coating in between the two paint layers allowed to perfectly remove the upper styrene-acrylic paint without affecting the layer below, operating with a fluence of approximately $2.60 \mathrm{~J} / \mathrm{cm}^{2}$ and $6 \mathrm{~Hz}$ frequency in LQS-II mode using water as wetting agent.

Different results were recorded in the mixed stratigraphies (sample C or D). A cleaning procedure based on the micro-swab application of a single solvent (e.g., propylene carbonate) generally gave quite good results in removing the graphical vandalism, although a loss of the glossy finish or a staining of the painting layer underneath was often observed, especially on the unprotected portion of the specimens. Any or very few paint remains were identified by FTIR and Py-GC/MS, and also color measurements showed no significant color changes of the base paint when propylene carbonate was used for cleaning, except for sample D_I_np where a $\Delta E$ value higher than the human eye threshold was found. For both specimens (i.e., C_I and D_I) the presence of the protective coating allowed to ease the cleaning and to better preserve the paint layer underneath, thus resulting in a $\Delta E$ value lower than 3.

Nanorestore Gels ${ }^{\circledR}$ may represent an alternative cleaning solution. In particular, a longer application time (up to $1 \mathrm{~h}$ ) was necessary for the removal of the red styrene-acrylic paint from the green alkyd layer underneath when the protective coating was absent (C_III_np), while in all other cases a 30-min application was sufficient for an effective removal. Nanorestore Gel ${ }^{\circledR}$ exhibited a good selectivity: after the cleaning treatment, no remains of the unwanted paint layer were detected by FTIR and Py-GC/MS analyses on sample C, while on sample D only few traces of alkyd spray paint 
were detected by Py-GC/MS. In both cases the protective layer was preserved and proved to facilitate obtaining good cleaning results, as confirmed also by the colorimetric measurements, which reported $\Delta E$ values slightly lower than the human eye threshold, unlike for the unprotected areas.

Regarding laser cleaning, although the results achieved on sample $C$ by using LQS-I in wet condition with a fluence of $4.14 \mathrm{~J} / \mathrm{cm}^{2}$ and frequency $7 \mathrm{~Hz}$ may be may be considered acceptable by visual assessment, the analytical campaign proved the presence of traces of unwanted paint. On sample $\mathrm{D}$, on the contrary, the laser treatment was not effective at all in removing the unwanted upper paint and the different selected parameters just provided thinning or an incomplete removal of the green alkyd layer.

\section{Conclusions}

It is absolutely clear that the cleaning operation still remains a challenging task in the conservation of street art as either the act of vandalism is chemically similar or different from the constituent materials of the mural involved. The fragile balance between the effectiveness and efficiency of the cleaning method is noticeable in each case study herein described. The use of a protective coating composed by a water-based mixture of reactive organosiloxane oligomers provided heterogeneous results in terms of effectiveness of the removal of unwanted paint materials without damaging the original paint layers. Despite this, in general the presence of a protective coating allows for better cleaning.

The use of a common methodology on four different stratigraphies of two paints confirmed that the interactions of the different layers deeply influenced the approach to the cleaning, obliging to evaluate the efficiency of a cleaning method on the basis of both the capability to remove the top "unwanted" layer and the absence of interactions with the bottom paint. In this perspective, the definition of shareable guidelines for the cleaning of street art murals should be based more on the identification of common protocols and approaches than on the formulation of ready-made methodologies and products. The experience gathered till now highlights that the statement of the main criteria of evaluation is a fundamental step, aimed to gear the set-up of mock-ups, representative of the real case studies, and to the definition of an effective analytical campaign. Due to the great variety of interactions observed, combined with the great scenario of painting materials, technique of execution and influence of the environment, the positive results of the preliminary tests on mock-ups should be then confirmed with further applications on the mural surface, before assessing the cleaning methodology, whose efficacy is absolutely case dependent.

Author Contributions: Conceptualization, D.S., M.B., P.C., A.S., and C.R.; methodology, A.S. and F.Z.; validation D.S., M.B., C.R., and P.C.; formal analysis, C.R., G.P., and M.B.; investigation, D.S., M.B., C.R., and P.C.; writing-original draft preparation, M.B. and C.R.; writing-review and editing, D.S. All authors have read and agreed to the published version of the manuscript.

Funding: This research was funded by the European Commission, Program Erasmus Plus, Key Action 2, Cooperation for innovation and the exchange of goo practices-Knowledge Alliance 2017, Project No. 588082-EPP-A-2017-1-IT-EPPKA-KA.

Acknowledgments: The authors are grateful to Laura D'Amico for the contribution given during her master's degree thesis.

Conflicts of Interest: The authors declare no conflict of interest.

\section{References}

1. Scalarone, D.; Bertasa, M.; Croveri, P.; Cardinali, M.; Stoisa, S.; Ricci, C.; Saccani, I.; Cavanna, G.; Bestetti, R.; Riggiardi, D.; et al. CAPuS project: Considerazioni sui supporti nella street art attraverso la voce degli artisti. In Proceedings of the VIII Congresso internazionale Colore e Conservazione, Venezia Mestre, Italy, 23-24 November 2018; Il Prato: Saonara, Italy, 2020; ISBN 978-88-6336-494-1.

2. Rava, A.; Collina, A. (Eds.) Dossier StreetArt—n. 109, Kermes-Restauro, Conservazione e Tutela del Patrimonio Culturale; Lexis Compagnia Editoriale: Torino, Italy, 2019; ISBN 978-88-32029-13-0. 
3. Chatzidakis, M. Street art conservation in Athens: Critical conservation in a time of crisis. Stud. Conserv. 2016, 61, 17-23. [CrossRef]

4. Shank, W.; Norris, D.H. Giving contemporary murals a longer life: The challenges for muralists and conservators. Stud. Conserv. 2008, 53, 12-16. [CrossRef]

5. Drescher, T.W. Priorities in Conserving Community Murals, Symposium. In Proceedings of the Mural Painting and Conservation in the Americas, Los Angeles, CA, USA, 16-17 May 2003.

6. Santabárbara, C. Street Art Conservation: Beyond Mural Restoration. In OPUS 2/2018, Varagnoli, C. Eds.; Gangemi: Rome, Italy, 2018; Volume 2, pp. 149-164, ISBN 978-88-4923-712-2.

7. Germinario, G.; Van Der Werf, I.D.; Sabbatini, L. Chemical characterisation of spray paints by a multi-analytical (Py/GC-MS, FTIR, $\mu$-Raman) approach. Microchem. J. 2016, 124, 929-939. [CrossRef]

8. La Nasa, J.; Orsini, S.; Degano, I.; Rava, A.; Modugno, F.; Colombini, M.P. A chemical study of organic materials in three murals by Keith Haring: A comparison of painting techniques. Microchem. J. 2016, 124, 940-948. [CrossRef]

9. Cucci, C.; Bartolozzi, G.; De Vita, M.; Marchiafava, V.; Picollo, M.; Casadio, F. The colors of keith haring: A spectroscopic study on the materials of the mural painting tuttomondo and on reference contemporary outdoor paints. Appl. Spectrosc. 2016, 70, 186-196. [CrossRef]

10. La Nasa, J.; Degano, I.; Modugno, F.; Colombini, M.P. Industrial alkyd resins: Characterization of pentaerythritol and phthalic acid esters using integrated mass spectrometry. Rapid Commun. Mass Spectrom. 2014, 29, 225-237. [CrossRef]

11. Di Crescenzo, M.M.; Zendri, E.; Sánchez-Pons, M.; Fuster-López, L.; Yusá-Marco, D.J. The use of waterborne paints in contemporary murals: Comparing the stability of vinyl, acrylic and styrene-acrylic formulations to outdoor weathering conditions. Polym. Degrad. Stab. 2014, 107, 285-293. [CrossRef]

12. Pintus, V.; Wei, S.; Schreiner, M. UV ageing studies: Evaluation of lightfastness declarations of commercial acrylic paints. Anal. Bioanal. Chem. 2011, 402, 1567-1584. [CrossRef]

13. Ploeger, R.; Scalarone, D.; Chiantore, O. The characterization of commercial artists' alkyd paints. J. Cult. Heritage 2008, 9, 412-419. [CrossRef]

14. Zieba-Palus, J. Examination of spray paints by the use of reflection technique of microinfrared spectroscopy. J. Mol. Struct. 2005, 229-234. [CrossRef]

15. Learner, T. Analysis of Modern Paints; The Getty Conservation Institute: Los Angeles, CA, USA, 2004.

16. Burns, D.T.; Doolan, K. A comparison of pyrolysis-gas chromatography-mass spectrometry and Fourier transform infrared spectroscopy for the analysis of a series of modified alkyd paint resins. Anal. Chim. Acta 2000, 422, 217-230. [CrossRef]

17. Lettieri, M.; Masieri, M. Surface characterization and effectiveness evaluation of anti-graffiti coatings on highly porous stone materials. Appl. Surf. Sci. 2014, 288, 466-477. [CrossRef]

18. Whitford, M.J. Getting Rid of Graffiti. A Practical Guide to Graffiti Removal and Anti-Graffiti Protection; E \& FN Spon: London, UK, 1992.

19. Nervo, M.; Piccirillo, A. Progetto degrado urbano. Vandalismo grafico e ragni tessitori, Cronache vol. 9; CELID: Torino, Italy, 2019; ISBN 8867891685.

20. Pozo-Antonio, J.S.; Rivas, T.; Jacobs, R.; Viles, H.; Carmona-Quiroga, P. Effectiveness of commercial anti-graffiti treatments in two granites of different texture and mineralogy. Prog. Org. Coat. 2018, 116, 70-82. [CrossRef]

21. Gomes, V.; Dionísio, A.; Pozo-Antonio, J.S. Conservation strategies against graffiti vandalism on Cultural Heritage stones: Protective coatings and cleaning methods. Prog. Org. Coat. 2017, 113, 90-109. [CrossRef]

22. Macchia, A.; Ruffolo, S.A.; Rivaroli, L.; Malagodi, M.; Licchelli, M.; Rovella, N.; Randazzo, L.; La Russa, M.F. Comparative study of protective coatings for the conservation of Urban Art. J. Cult. Heritage 2020, 41, 232-237. [CrossRef]

23. Sanmartín, P.; Bosch-Roig, P. Biocleaning to remove graffiti: A real possibility? Advances towards a complete protocol of action. Coatings 2019, 9, 104. [CrossRef]

24. Sanmartín, P.; DeAraujo, A.; VasanthaKumar, A.; Mitchell, R. Feasibility study involving the search for natural strains of microorganisms capable of degrading graffiti from heritage materials. Int. Biodeterior. Biodegradation 2015, 103, 186-190. [CrossRef]

25. Giacomucci, L.; Toja, F.; Sanmartín, P.; Toniolo, L.; Prieto, B.; Villa, F.; Cappitelli, F. Degradation of nitrocellulose-based paint by desulfovibrio desulfuricans ATCC 13541. Biodegradation 2012, 23, 705-716. [CrossRef] 
26. Sanmartín, P.; Cappitelli, F.; Mitchell, R. Current methods of graffiti removal: A review. Constr. Build. Mater. 2014, 71, 363-374. [CrossRef]

27. Prati, S.; Sciutto, G.; Volpi, F.; Rehorn, C.; Vurro, R.; Blümich, B.; Mazzocchetti, L.; Giorgini, L.; Samorì, C.; Galletti, P.; et al. Cleaning oil paintings: NMR relaxometry and SPME to evaluate the effects of green solvents and innovative green gels. New J. Chem. 2019, 43, 8229-8238. [CrossRef]

28. Ferrari, P.; Chelazzi, D.; Bonelli, N.; Mirabile, A.; Giorgi, R.; Baglioni, P. Alkyl carbonate solvents confined in poly (ethyl methacrylate) organogels for the removal of pressure sensitive tapes (PSTs) from contemporary drawings. J. Cult. Herit. 2018, 34, 227-236. [CrossRef]

29. Samorì, C.; Galletti, P.; Giorgini, L.; Mazzeo, R.; Mazzocchetti, L.; Prati, S.; Sciutto, G.; Volpi, F.; Tagliavini, E. The green attitude in art conservation: Polyhydroxybutyrate-based gels for the cleaning of oil paintings. ChemistrySelect 2016, 1, 4502-4508. [CrossRef]

30. Sousa, M.; Melo, M.J.; Casimiro, T.; Aguiar-Ricardo, A. The art of $\mathrm{CO}_{2}$ for art conservation: A green approach to antique textile cleaning. Green Chem. 2007, 9, 943. [CrossRef]

31. Spepi, A.; Pizzimenti, S.; Duce, C.; Vozzi, G.; De Maria, C.; Tiné, M.R. Chemico-physical characterization and evaluation of coating properties of two commercial organosilicons. J. Therm. Anal. Calorim. 2019, 138, 3277-3285. [CrossRef]

32. Ormsby, B.; Learner, T.; Schilling, M.; Druzik, J.; Khanjian, H.; Carson, D.; Foster, G.; Sloan, M. The Effects of Surface Cleaning on Acrylic Emulsion Paintings: A Preliminary Investigation. Available online: https://www.tate.org.uk/research/publications/tate-papers/06/effects-of-surface-cleaningon-acrylic-emulsion-painting-preliminary-investigation (accessed on 22 October 2020).

33. Ricci, C.; Gambino, F.; Nervo, M.; Piccirillo, A.; Scarcella, A.; Zenucchini, F.; Ramil, A.; Pozo-Antonio, J. Enhancement of graffiti removal from heritage stone by combining laser ablation and application of a solvent mixture. Constr. Build. Mater. 2020, 262, 119934. [CrossRef]

34. Schanda, J. Colorimetry, Wiley-Interscience; John Wiley \& Sons: Hoboken, NJ, USA, 2007; pp. 61-63, ISBN 978-0-470-04904-4.

35. Witzel, R.F.; Burnham, R.W.; Onley, J.W. Threshold and suprathreshold perceptual color differences. J. Opt. Soc. Am. 1973, 63, 615-625. [CrossRef]

36. Van Gorkum, R.; Bouwman, E. The oxidative drying of alkyd paint catalysed by metal complexes. Co-ord. Chem. Rev. 2005, 249, 1709-1728. [CrossRef]

Publisher's Note: MDPI stays neutral with regard to jurisdictional claims in published maps and institutional affiliations.

(C) 2020 by the authors. Licensee MDPI, Basel, Switzerland. This article is an open access article distributed under the terms and conditions of the Creative Commons Attribution (CC BY) license (http://creativecommons.org/licenses/by/4.0/). 